\title{
Distribution of stoneflies of the family Taeniopterygidae (Plecoptera) in the Czech Republic: earlier data, new records and recent distributional changes
}

\author{
Jindřiška Bojková, Tomáš Soldán, Jan Špaček \& Michal Straka
}

\begin{abstract}
Distribution of stoneflies of the family Taeniopterygidae (Plecoptera) in the Czech Republic: earlier data, new records and recent distributional changes. - Čas. Slez. Muz. Opava (A), 60: 239-258, 2011.

Abstract: Altogether 14 species of Taeniopterygidae were previously mentioned from the Czech Republic; 11 species were listed in check-list of Plecoptera of Czechoslovakia, three species were recorded recently. Main aims of this study were to summarize all known distributional data, reexamine available material, classify species to the categories of threat based on new distributional data, and discuss all species ever supposed to occur in the Czech Republic. In total eight species were found to occur in the Czech Republic with certainty. Two species, Brachyptera seticornis and B. risi, are common in the Czech Republic, the latter exhibiting some expansion in Bohemia at present. Critically endangered Brachyptera braueri missing in the past 40 years has been recently found in several rivers showing some recovery of the original area. Endangered Taeniopteryx nebulosa exhibited considerable decline in lowland and colline streams. New records are presented for near threatened montane species B. starmachi, endangered B. monilicornis, newly classified endangered $T$. auberti, and vulnerable Taeniopteryx hubaulti. No reliable data have been found as to distribution of Brachyptera trifasciata, Taeniopteryx kuehtreiberi, T. araneoides, and Oemopteryx loewii, respective earlier records were based on misidentification. The occurrence of Rhabdiopteryx acuminata and $R$. neglecta is doubtful at this moment, because it was based on the problematic determination of larval material. These species cannot be formally considered a part of the Czech fauna due to lack of voucher material. Possible occurrence of other Rhabdiopteryx species showing the area adjacent to the Czech Republic is discussed.
\end{abstract}

Key words: Plecoptera, Taeniopterygidae, historical and earlier data, new area records, distribution, habitats, species protection

\section{Introduction}

Although more than 100 species of stoneflies (Plecoptera) are currently supposed to occur in the Czech Republic (cf. Soldán et al. 1998, Helešic et al. 2005, Bojková 2009) any modern species check-list actually does not exist. The only published list of Plecoptera of the former Czechoslovakia enumerated 90 species known in Bohemia and Moravia (81 species in Bohemia and 75 species in Moravia; Raušer 1977). This list appeared a good many years ago and, despite a number of papers aimed at ecology and occurrence of some rare species published in the course of past decades, a good deal of data have to be re-examined or need a revision. Furthermore, the list contains several species whose occurence in individual regions of Czechoslovakia was apparently only tentative, supposed by the author. Not all of these assumptions were later confirmed by concrete finding of species. Naturally, there is also problem to differentiate between the doubtful occurrence and supposed occurrence, since both are marked by "?" in the list.

Within the order Plecoptera, Taeniopterygidae are definitively not an exception. The occurrence of some species has been apparently tentative and numerous misidentifications have tended to further confusion(s) in distributional data. Moreover, some species have been mentioned more than a century ago and data on the actual present distribution are missing. In the only existing check-list by Raušer (1977), the following 11 species of the family Taeniopterygidae are presented $(\mathrm{B}=$ Bohemia, approximately the Labe [Elbe] river basin; $\mathrm{M}$ = Moravia, approximately the Dunaj [Danube] river basin); the question mark means doubtful 
and/or tentative occurrence): Taeniopteryx auberti (?B), T. araneoides (?B), T. hubaulti (B, $\mathrm{M})$, T. kuehtreiberi (B), T. nebulosa (B, M); Brachyptera braueri (B, M), B. monilicornis (B, M), B. risi (B, M), B. seticornis (B, M), B. trifasciata (B), Oemopteryx loewii (?B); no species of the genus Rhabdiopteryx is reported from Bohemia and Moravia. Since then, the occurrence of an additional species, namely B. starmachi, has been proved (Bojková 2009). Working-list of Plecoptera species of the Labe, Morava and Odra rivers compiled by Soldán et al. (1998) is based partly on presumable distribution and contains even 16 species (besides the species mentioned above, also Taeniopteryx schoenemundi, Rhabdiopteryx acuminata, Rh. alpina, and Rh. neglecta).

The objective of the present paper is to: (i) prove or control (if possible) and summarize earlier data on the occurrence of individual representatives of the Taeniopterygidae in the Czech Republic, (ii) present new findings emphasizing new area records, (iii) discuss and/or correct possible misidentification and earlier confusing data and to re-examine earlier material, if available, (iv) discuss present areas as well as changes in distribution with respect to species ecological requirements, and (v) correct existing red-list of Plecoptera of the Czech Republic in order to include missing species and remove the species actually not occurring in this area.

\section{Material and Methods}

The material examined (preserved in $70 \%$ ethanol) is currently deposited in four collections: the collection of the Department of Entomology, National Museum, Prague (NMPC), Jindřiška Bojková's collection at the Department of Botany and Zoology, Masaryk University, Brno, Czech Republic (JBBC), Michal Straka's collection, Hustopeče, Czech Republic (MSHC), and Jan Špaček's collection, Hradec Králové, Czech Republic (JSHKC).

The historical material from the study area collected by Jaroslav Raušer is deposited in the Jaroslav Raušer's collection at the Department of Botany and Zoology, Masaryk University, Brno, Czech Republic (JRBC). The material was re-identified by the senior author (J. Bojková) within the project of revision of the Křelinová's and Raušer's historical collections (see Bojková 2009 for details). Although the material of Brachyptera seticornis and $B$. risi is missing and thus cannot be revised, the records were included to the text and grid maps, since at most places these species occur till the present and do not show any pronounced changes in distribution and occurrence. These records were registered in the unpublished protocols which are deposited at the Department of Botany and Zoology, Masaryk University, Brno, Czech Republic.

The system and nomenclature of the group follow Graf et al. (2002). Distribution and ecological requirements refer to Illies (1978), Zhiltzova (2003), Graf et al. (2009), and Teslenko \& Zhiltzova (2009), other sources are given in the text.

New distributional records are presented in the following sequence: name of stream or water body, name of adjacent settlement, elevation above sea level, (code of the map field), date of collection, number and life cycle stage of material examined (abbreviations: $\widehat{\partial}$ - male,,+ - female, I - imago, L - larva, E - exuvia), name(s) of collector(s), name of determining and/or revising author, and collection where the material is deposited. The code number (in parentheses) refers to the map field of the Central European grid for mapping flora and fauna (Ehrendorfer \& Hamann 1965, adapted by Novák 1989 and Pruner \& Míka 1996). Records were arranged according code numbers. Records were not listed in detail in Brachyptera seticornis. This species is very common in montane and submontane streams all over the Czech Republic and detailed enumeration of individual records is not necessary. Details on published records can be found in numerous literature sources cited in the respective paragraph.

Published records are presented by name of stream and adjacent settlement, and respective reference. Several historical records by Klapálek are presented only by the name of settlement, as detail information on the location of records is not available.

Status and categories from the species protection point of view are based on preliminary protection list of the Šumava Mts. species by Soldán (2004) and list by Soldán et al. (1998) but mainly on that by Helešic et al. (2005) in the Red List of Threatened Invertebrates of the Czech Republic (Farkač et al. 2005) comprising altogether 12 species of the family Teaniopterygidae. Of these, 4 species are included in the category regionally extinct (RE), 3 critically endangered (CR), 2 endangered (EN), 2 vulnerable (VU), and 1 species near threatened (NT); Taeniopteryx auberti has not been treated. 


\section{Results and discussion}

\section{RECORDS ON INDIVIDUAL SPECIES OF TAENIOPTERYGIDAE}

\section{Brachyptera braueri (KLAPÁLEK, 1900)}

(Fig. 1)

Published records: Vltava river in Prague at Císařská louka, Závist and Jarov; Nežárka river in Veselí nad Lužnicí; Plzeň (Klapálek 1905); Hnidousy; Hradec Hrálové (Klapálek 1906); Praha-Krč (coll. Klapálek); Oslava river in Oslavany (Adámek 1972, Sukop \& Spurný 2003); Berounka river at Radotín and Zvíkovec (Bojková \& Špaček 2006). Raušer (1992) stated that the species was known from the Morava river near Olomouc in the past. Other records refer to montane streams where the occurrence of B. braueri is improbable (see the section Distribution and habitat): small montane brooks Jatný, Poledněna, Mazák, and Sepetný potok in the Moravskoslezské Beskydy Mts. (Zelinka 1950); Jizera river in Velká Jizerská Louka (Křelinová 1962; this record was mentioned also in Winkler 1977). Only material from Jizera river is available in the Křrelinová's collection in the National Museum in Prague. It was re-identified as B. starmachi (see below).

New Records: Labe river near Děčín, Podskalí, 120 m (5151), 8.iii.2011, 1 đ̊I, M. Straka leg. et det. (MSHC); Vltava river in Prague, 190 m (5952), 7.v.1944, 1 Ĵ, 2 우 I, V. Landa leg., J. Bojková det. (NMPC); Mže river above Milíkov, 400 m (6243), 29.xii.2006, 4 L, J. Špaček leg. et det. (JSHKC); Mže river below Milíkov, 380 m

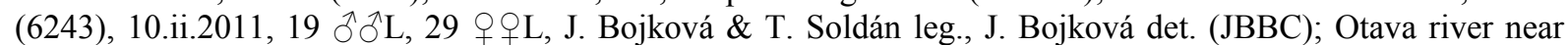

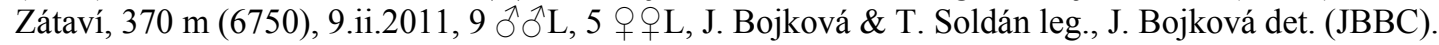

Distribution and habitat: European species with area extension to western Siberia (e.g. Komi autonomous republic of Russia), missing in the British Islands, Apennine Peninsula, western part of Balkans and north part of Scandinavia. It occurs predominantly in hyporhitral and epipotamal rivers in coline and lowland zones.

Remarks: The species dramatically declined during the second half of the 20th century all over Europe and became a rarity with scattered distribution (Zwick 1992, Fochetti \& Tierno de Figueroa 2006). In the Czech Republic, it seems that it was common species of lowland rivers in the past. According to Klapálek (1905) it was the most common stonefly in Prague that can be found on the walls of houses near the Vltava River. It was trivially named "Prague fly" by raftsmen and fishermen thanks to its mass occurrence on bridges and quays (Obenberger 1958). Imagines were collected by V. Landa in the Vltava river in Prague in 1944 for the last time. Personal opinion of Prof. Landa (Landa et al. 1997) was that, despite of disappearing of the mass occurrence of the species around 1945, some individuals could be observed each spring till 1950 but in rapidly decreasing numbers. This corresponds with the results of Krrelinová (1962) who did not find any individual in Prague in 1960 and those of Soldán et al. (1998) who failed to collect any speciment in 1990-1995. Decline of B. braueri was probably caused by a strong pollution in the 1960-1990's (e.g., in the Berounka river) or by construction of reservoirs (the Vltava river). In the latter case, populations at localities in Prague managed to survive upstream construction of the Vrané (1935) and Štěchovice (1944) reservoirs. However, installation of the Slapy (1955), Kamýk (1961) and Orlík (1962) became probably fatal. The species was found to be endangered by Raušer (1992) and even critically endangered by Helešic et al. (2005) due to lack of any recent record. Larvae of B. braueri started to be found again as late as in 2003-2005 in the Berounka river (Bojková \& Špaček 2006). At the same time the species was found in several rivers in Germany, in the Werra river and Saale river in Thüringen (Brettfeld \& Bellstedt 2000, 2003), the Isar river in Bavaria (Dorn \& Weinzierl 2003), and the Mulde/Elbe river in Saxony and Saxony-Anhalt (Hohmann 2004). Following investigation showed the species is relatively common in Central Germany, not far from the Czech Republic (Küttner et al. 2008). New records from the Mže river, Otava river and even from the Labe river near Děčín presented here showed promising trend towards a recovery of the species area also in the Czech Republic. 


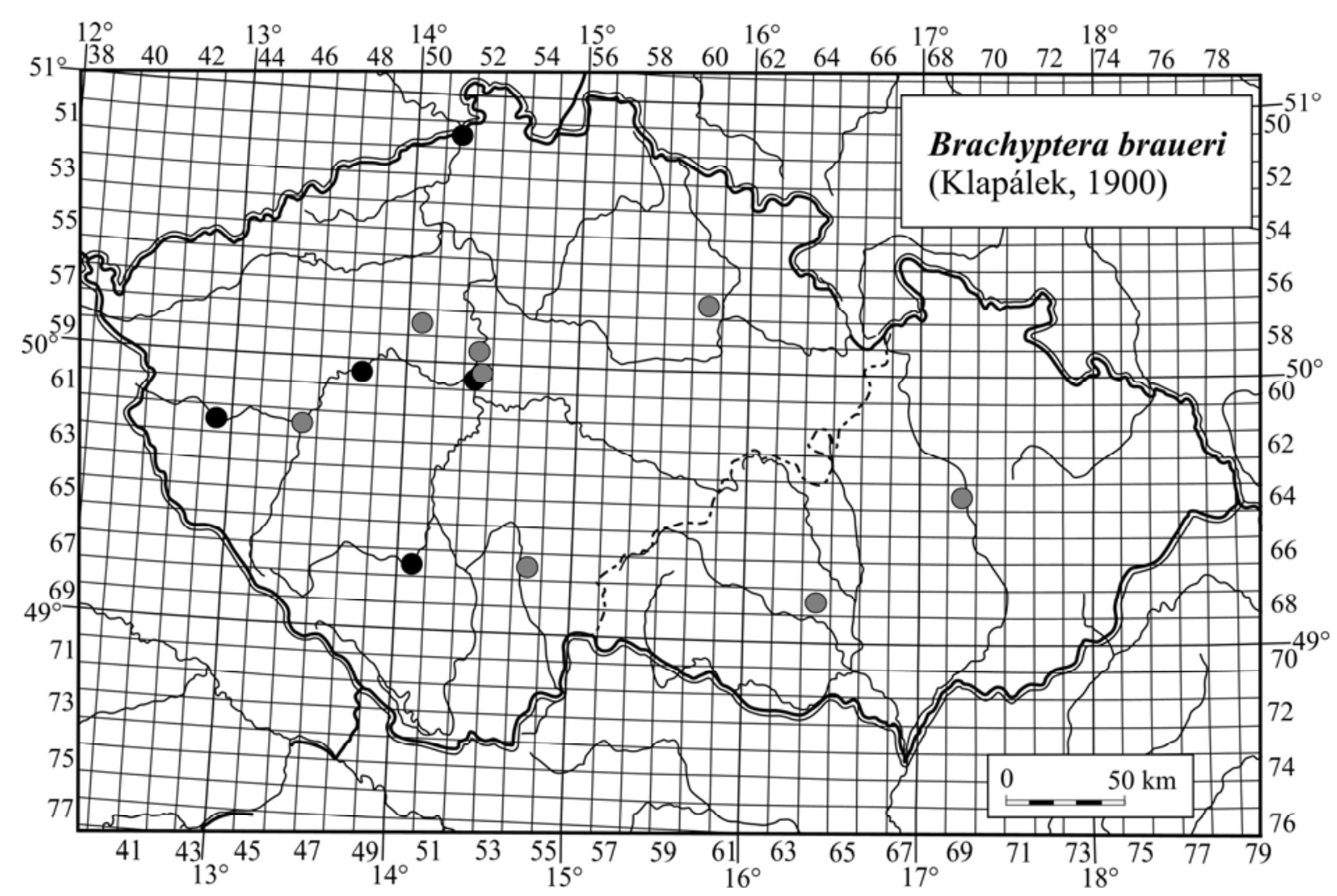

Fig. 1: Distribution of Brachyptera braueri. Grey circles indicate historic occurrences, black circles present records.

\section{Brachyptera monilicornis PICTET, 1841}

(Fig. 2)

Published records: Havlíčkův (formerly Německý) Brod and Libice (Klapálek 1903, 1906; sub Taeniopteryx kempnyi); Moravice river near Jamartice, at Karlov, Malá Morávka, Velká Št’áhle, Břidličná and Valšov, and Podolský potok brook above Janovice in the Hrubý Jeseník Mts. (Losos \& Marvan 1957); estuary of Náhlovský potok brook, Frymburk nad Vltavou, Vltava bellow the Lipno II impoundment, Vyšší Brod (Soldán 2004, imagines collected on snow).

New records: Blanice river near Blažejovice, 760 m (7049), 18.ii.2010, 10 L; Blanice river at Myšenec, 380 m (6751), 9.ii.2011, 1 §̌L, J. Bojková \& T. Soldán leg., J. Bojková det. (JBBC).

Distribution and habitat: Submediterranean European species, occurring from the Iberian and Apennine Peninsula (Ravizza \& Fochetti 1999, Tierno de Figueroa et al. 2003), over the Alps (e.g. Graf 1999, Reusch \& Weinzierl 1999), Central and Western Highlands (e.g. Reusch \& Weinzierl 1999, Soldán 2004, Le Doaré \& Vinçon 2006) to the Carpathians and western part of Balkan (e.g. Kis 1974, Zhiltzova 2003, Murányi 2008). Larvae inhabit predominantly hyporhitral and epipotamal streams in submontane and coline zones (Graf et al. 2009, Ravizza \& Fochetti 1999).

Remarks: B. monilicornis is an inhabitant of hyporhitral and epipotamal streams which became very restricted and rare in Central Europe, inferring from the historic data from the first half of the 20th century (Zwick 1992). Recently it was found at numerous localities in Upper Austria, these findings may indicate a certain recolonisation (Malicky-Ruzicka \& Malicky 2003). Our recent records from the streams in foothills of the Šumava (Bohemian Forest) Mts. are situated close to this region but the species seems to be solitary or rare. The species is considered endangered in the Czech Republic (Helešic et al. 2005) but it would deserve rather "data deficient" category of species protection owing to still fragmentary data on its distribution. 


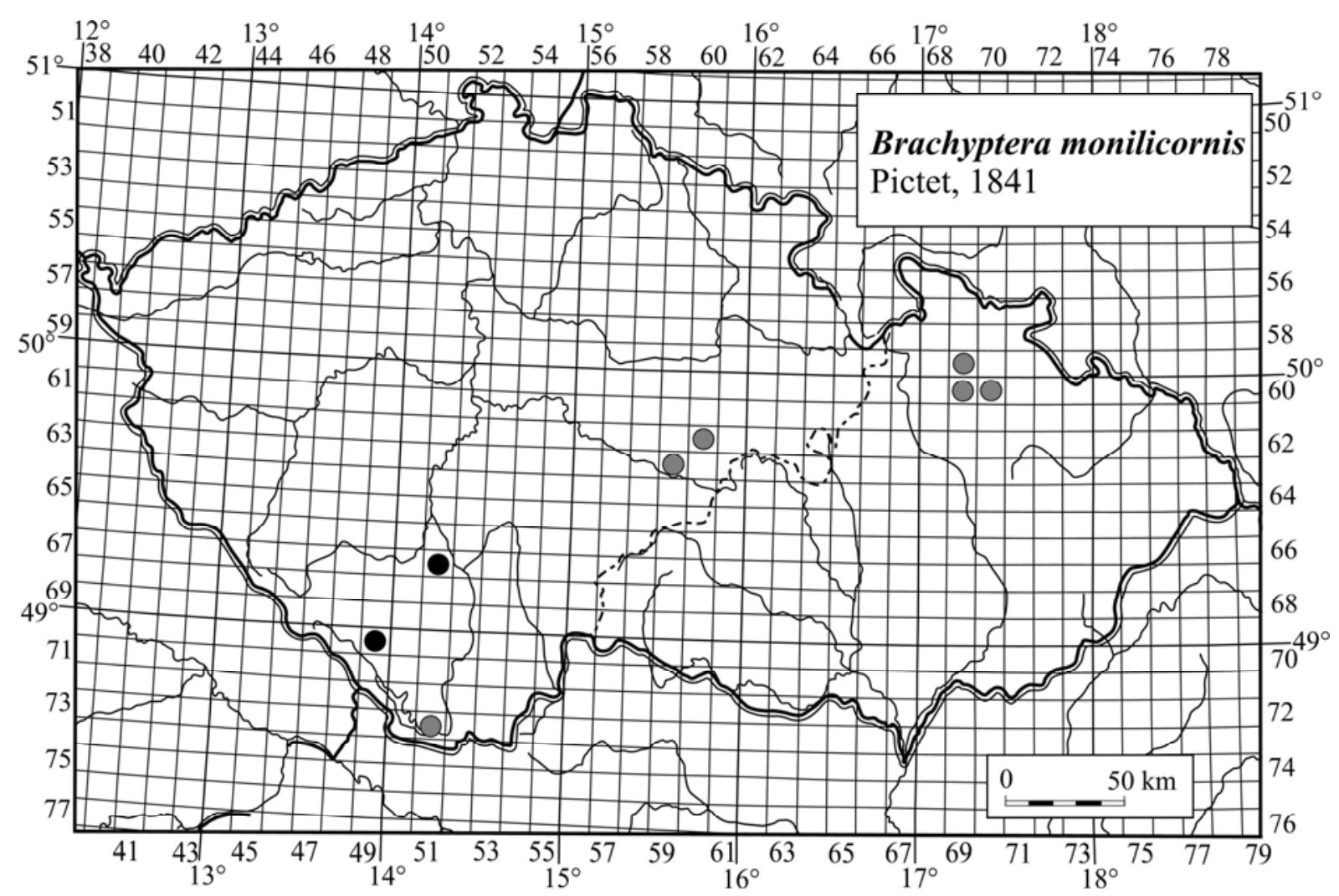

Fig. 2: Distribution of Brachyptera monilicornis. Grey circles indicate published records, black circles new records.

\section{Brachyptera risi (Morton, 1896)}

(Fig. 3)

Published records: Vůznice, Budislav, Německý (Havlíčkův) Brod, Libice (Klapálek 1905); Turnov, Poděbrady, Hranice (coll. Klapálek); Schwarzbach, Mlýnský potok brook, and Weitfäldský potok brook in the Šumava Mts. (Šámal 1920); Černá Ostravice brook below the Bílý Kř́̌́z Mt. (Hrabě et al. 1954); Moravice river at Karlov and Břidličná (Losos \& Marvan 1957); eight localities in the Vsetínská Bečva river basin: unnamed brooks at Bařina, Podt'atý potok brook at Podt'até, Stanovnice brook above Karolinka, Jasenka brook above Horní Jasenka, Ratibořka brook above Ratiboř, unnamed brooks at Jablůnka, Vsetínská Bečva river above Jablůnka and at Jarcová (Švec 1960); Stř́brný potok brook at Nýznerov in the Rychlebské hory Mts. (Obrdlík 1978, Tuša 2001); Šumný potok brook above Bělá pod Pradědem in the Hrubý Jeseník Mts. (Obrdlík 1981); Hájený potok brook in the Jizerské hory Mts. (Preisler \& Špaček 2001); Keblanský potok brook near Buškův Hamr and Černá river at Černé údolí in the Novohradské hory Mts. (Soldán \& Špaček 2002); Bílý potok brook above Bílá voda, Kotelný potok brook above Karlov, and Červený potok at Vidnava in the Jeseníky Mts., tributary of Březná river below Jedlí in the Zábřežská vrchovina, Brodecký potok brook above Čunín and Divoký potok in the Drahanská vrchovina Mts., Kopytná brook above Milíkov, Sepetný potok brook and Mazák brook near Ostravice and unnamed brooks above Bílá in the Moravskoslezské Beskydy Mts., and brooks at Bukovec Nature Reserve in the Jablunkovské mezihoří (Tuša 2001); four localities in the České Švýcarsko National Park: Křinice river at Zadní Doubice, Brtnický potok at Zadní Doubice, unnamed brook at Na Tokáni, Doubický potok at Doubice (Bojková et al. 2010); 16 localities in the Bílé Karpaty Mts: Lubná brook near Korytná; Velička stream near Strání; left tributary of Kamenný potok brook, Strání; left tributary of Velička river, Javorník nad Veličkou; Járkovec brook near Kněždub; Radějovka brook above Radějov; Sudoměřický potok brook above Mlýnky; Mandát stram near Radějov u Strážnice; Rybnický potok brook near Javorník nad Veličkou; Zápechová stream near Nedašova Lhota; Hrušovka brook near Nedašov; Vlárka stream above Sidonie; Krátkovský potok brook, Vápenice u Starého Hrozenkova; Hrnčarův potok brook above Vápenice u Starého Hrozenkova; Hrubár brook above Lopeník; right tributary of Klanečnice stream near Strání (Bojková et al. 2011).

Unpublished historical records: Kudlovický potok brook above Kudlovice, Radějovka brook above Radějov, Olšava river above Pitín, Ráztoka brook at Rusava, Kýchová brook above Huslenky (Raušer 1958, unpublished protocols), Porubka brook above Dolní Lhota (Raušer 1956, unpublished protocols), unnamed brook near Beranovec (Raušer 1957, unpublished protocols). 
New records: Smědá river at Luh, 340 m (5156), 22.iv.2004, 1 L, V. Havlíček leg., J. Špaček det. (JSHKC); Smědá river at Bílý potok, $500 \mathrm{~m}$ (5157), 30.v.2008, 22 L; Rašovka brook at Český Dub, $310 \mathrm{~m}$ (5355), 27.iv.2006, 54 L; Oharka brook near Libíč, 320 m (5356), 22.iv.2004, 10 L; Kamenice river near Spálov, 290 m (5357), 2.iv.2007, 12 L, all J. Špaček leg. et det. (JSHKC); unnamed brook near Sklenařice, 490 m (5358),

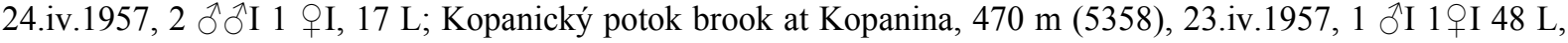
all Křelinová leg., J. Bojková det. (NMPC), 4.v.2011, 20 L, J. Bojková \& T. Soldán leg., J. Bojková det. (JBBC); Jizera river at Horní Sytová, 390 m (5358), 2.iv.2007, 6 L; Jizerka at Víchová nad Jizerou, 400 m (5358), 27.iv.2010, 48 L, all V. Havlíček leg., J. Špaček det. (JSHKC); Vápenický potok brook near airport in Vrchlabí, $480 \mathrm{~m}$ (5359), 8.iii.2007, 5 L; Bolkovský potok brook at Bolkov, 580 m (5360), 4.iii.2008, 1 L; Janovický potok brook above Janovice, 470 m (5360), 5.iii.2008, 2 L; Zlatý potok brook near Sklenařice, 580 m (5360), 1.v.1998, $11 \mathrm{~L}$; Černý potok brook near Kralovec, $520 \mathrm{~m}$ (5361), 1.v.2009, 8 L; left tributary of Heřmánkovický potok brook above Heřmánkovice, 500 m (5363), 3.iv.2008, 2 L; Veselka brook near Ktová, 280 m (5457), 21.iv.2005, 1 L; Lázeňský potok brook above Leopold, 450 m (5460), 8.v.1997, 16 L; Luční potok brook at Rudník, 390 m (5460), 4.iv.2008, 1 ๆ I, all J. Špaček leg. et det. (JSHKC); Malé Labe river at Prosečné, $390 \mathrm{~m}$ (5460),

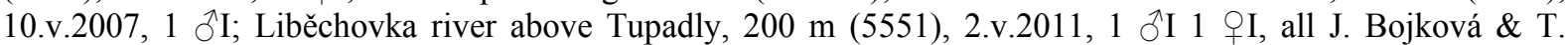
Soldán leg., J. Bojková det. (JBBC); Metuje river near Běloves, 350 m (5563), 6.iv.2011, 1 L, V. Havlíček leg., J. Špaček det. (JSHKC); Pasecký potok brook above Bílá voda, 500 m (5567), 28.iv.2004, 1 L; Rolava river at Rolava, 900 m (5641), 1.iv.2004, 2 ỗ 1 q I 43 L, all J. Špaček leg. et det. (JSHKC); Rolava river near Nové Hamry, 770 m (5642), 28.v.2008, 18 L, J. Bojková \& T. Soldán leg., J. Bojková det. (JBBC); Valovický potok brook at Velký Třebešov, 270 m (5662), 3.iv.2008, 38 L; Olešenka river at Peklo, 330 m (5663), 10.iv.2009, 33 L, J. Špaček leg. et det. (JSHKC); Lomnice river at Kyselka, 420 m (5744), 26.v.1955, 1 đII, Křelinová leg., J. Bojková det. (NMPC), 4.v.2007, 1 L, J. Bojková \& T. Soldán leg., J. Bojková det. (JBBC); Zlatý potok near České Meziř́ííí, 260 m (5762), 6.iv.2004, 1 L, V. Havlíček leg., J. Špaček det. (JSHKC); Dědina brook near České Meziřričí, 260 m (5762), 17.v.2000, 1 \&I; Dědina brook near Chábory, 300 m (5763), 22.iv.2002, 1 JI 1 OI; Bělá river at Růženina Hut', 430 m (5763), 12.v.1998, 1 q, all P. Chvojka leg., J. Bojková det. (NMPC); Ramzovský potok brook near Ramzová, 630 m (5768), 20.v.2006, 1 L, J. Bojková \& S. Zahrádková leg., J. Bojková det. (JBBC); Stř́ibrný potok above Nýznerov, 490 m (5768), 28.iv.2004, 1 L, J. Špaček leg. et det. (JSHKC); Čistý potok brook near Třídomí, 600 m (5842), 4.vi.1993, 1 † I, P. Chvojka leg., J. Bojková det. (NMPC); Mlýnecký potok brook near Valeč, 550 m (5845), 21.iv.1955, 6 L, Křelinová leg., J. Bojková det. (NMPC); Zdobnice river in Vamberk, 320 m (5863), 2.v.2006, 6 L; Orličský potok brook above Pastviny, $480 \mathrm{~m}$ (5865), 9.v.2006, 55 L, all J. Špaček leg. et det. (JSHKC); Střela river at Pečkovice, 560 m (5944), 20.iv.1955, 1 I; Křelinová leg., J. Bojková det. (NMPC), 4.v.2007, 1 qI, J. Bojková \& T. Soldán leg., J. Bojková det. (JBBC); unnamed small brook at Pečkovice, 570 m (5944), 28.v.2008, 1 †I, J. Bojková \& T. Soldán leg., J. Bojková det. (JBBC); Šembera brook near Doubravčice, 255 m (5954), 27.vi.1955, 7 đ઼đI, Křelinová leg., J. Bojková det. (NMPC), 23.iv.2008, 1 qI 1 L, J. Bojková \& T. Soldán leg., J. Bojková det. (JBBC); Tichá Orlice river above Jablonné nad Orlicí, 420 m (5965), 2.v.2006, 18 L, V. Havlíček leg., J. Špaček det. (JSHKC); Pottův potok brook above Mariánské lázně, 650 m (6042), 10.iv.1999, 1 L, J. Špaček leg. et det. (JSHKC); Žlubinecký potok brook at Nižbor, 230 m (6050), 8.iv.2010, 1 L, J. Bojková \& T. Soldán leg., J. Bojková det. (JBBC); Klejnárka river near Malín, 210 m (6057), 16.iv.2008, 2 L, J. Bojková \& T. Soldán leg., J. Bojková det. (JBBC);

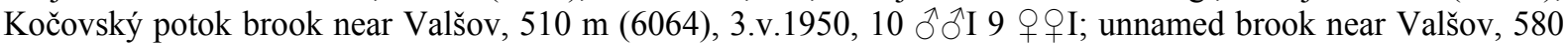

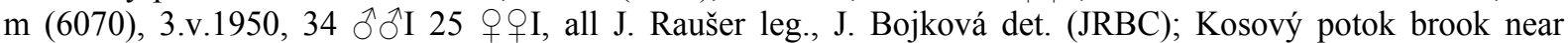
Černošín, 430 m (6143), 11.iv.2004, 2 L, J. Špaček leg. et det. (JSHKC); Voznický potok brook at Voznice, 380 m (6151), 7.iv.2010, 2 L, J. Bojková \& T. Soldán leg., J. Bojková det. (JBBC); Paběnický potok brook below Hraběšín, 300 m (6157), 13.iv.2007, 1 JI 2 O I I 8 L; Jánský potok brook near Březí, 360 m (6158), 27.iv.1956, 15 L; Váhanka brook near Vrběšice, 650 m (6159), 10.iv.2008, 2 L, all J. Špaček leg. et det. (JSHKC); Chrudimka river below Seč, 445 m (6160), 13.v.2010, 1 đ̊I, J. Bojková \& T. Soldán leg., J. Bojková det. (JBBC); Krounka river near Doly, 310 m (6162), 24.iv.2009, 148 L; Martinický potok brook near Perálec, 440 m (6162), 11.iv.2008, 190 L, all J. Špaček leg. et det. (JSHKC); Porubka brook near Dolní Lhota, 240 m (6174), 13.iv.2011, 2 L; Otročínský potok brook below Milíkov, 400 m (6243), 4.iv.2007, 1 L; Losinský potok brook near Kácov, 330 m (6256), 13.v.2010, 1 †I; Zbizubský potok brook near Kácov, 320 m (6256), 6.iv. 2010, 1 L, all J. Bojková \& T. Soldán leg., J. Bojková det. (JBBC); Cerhovka brook near Bezděkov, 430 m (6260), 15.iv.2004, 19 L; Chrudimka river near Hamry, 600 m (6261), 14.iv.2008, 8 L, all J. Špaček leg. et det. (JSHKC); Brodec brook near Světlá, 370 m (6355), 10.iv. 2007, 1 đI 1 L, J. Bojková \& T. Soldán leg., J. Bojková det. (JBBC); Bystřice river at Hlubočky, 305 m (6370), 25.iv.2007, 1 ठ઼' I; Trusovický potok brook

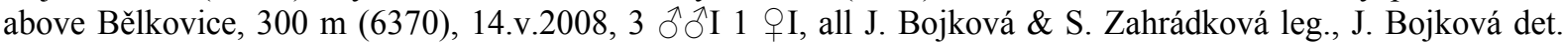
(JBBC); Hluchová river at Nýdek-Kolibiska, 560 m (6378), 1.v.2005, 1 §̊I, J. Bojková leg. et det. (JBBC); Olše river in Třinec, 310 m (6378), 16.iv.2007, 2 L, J. Bojková \& S. Zahrádková leg., J. Bojková det. (JBBC); Kopytná brook at Košařiska, 640 m (6477), 9.vi.2006, 1 ठI 3 우 I; Sihelský potok brook at Visalaje, $730 \mathrm{~m}$

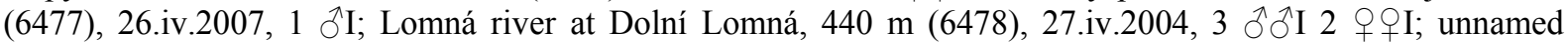
springbrook at Bukovec, 510 m (6479), 11.v.2006, 1 † I, all J. Bojková leg. et det. (JBBC); Trnava river near 


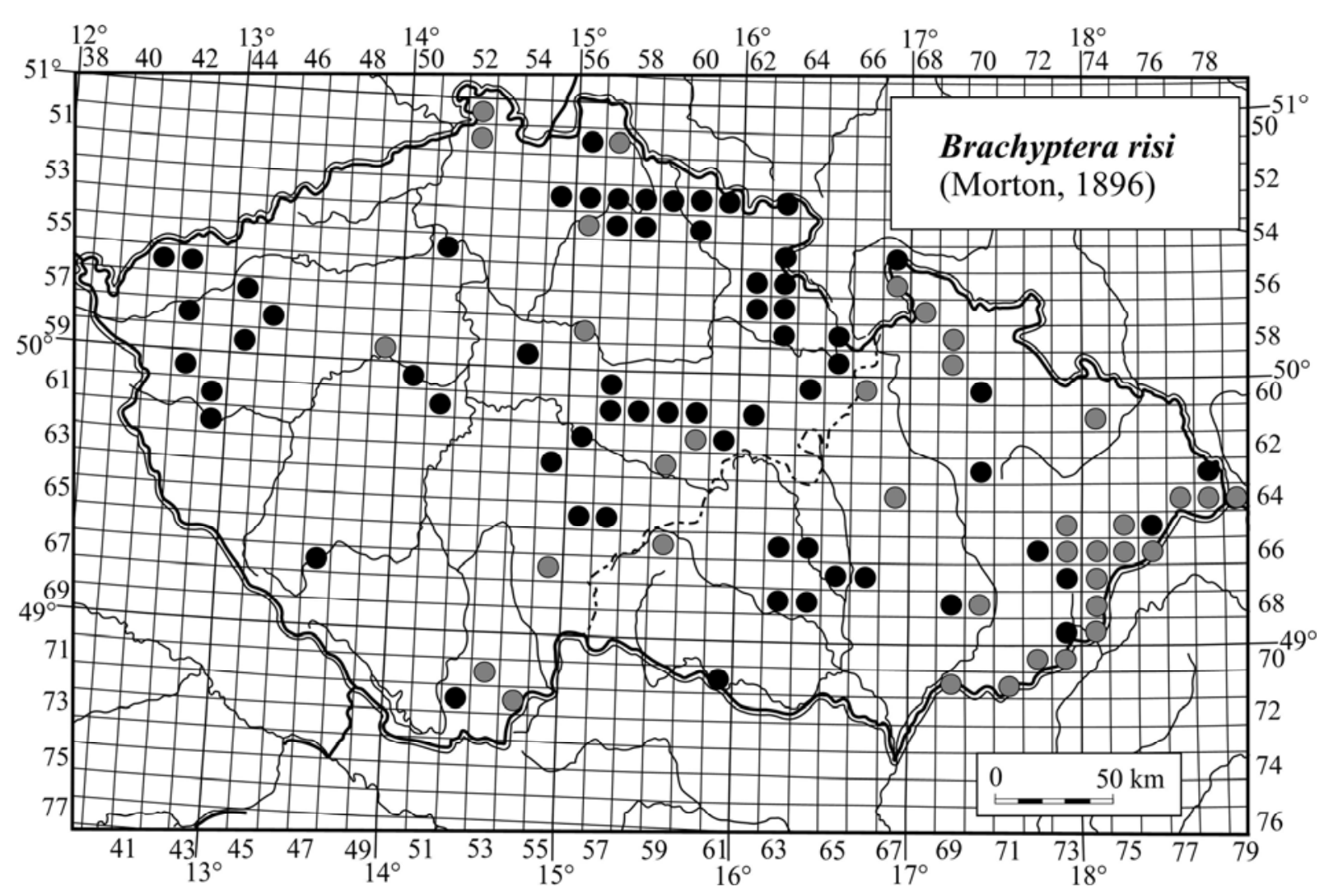

Fig. 3: Distribution of Brachyptera risi. Grey circles indicate published records, black circles new records.

Hrádek, 480 m (6556), 10.iv.2007, 5 L; Želivka river near Hodějovice, 490 m (6557), 23.iv.2008, 1 ๆ, all J. Bojková \& T. Soldán leg., J. Bojková det. (JBBC); Nedvědička river at Pernštejn, 370 m (6563), 19.iii.2007, 1 L, J. Bojková \& S. Zahrádková leg., J. Bojková det. (JBBC); Křeptovský potok brook near Běleč, 360 m (6564),

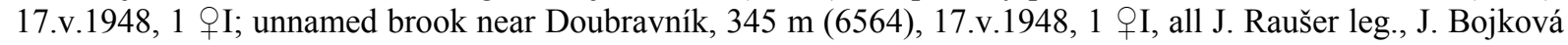
det. (JRBC); Rožnovská Bečva river at Horní Bečva, 570 m (6576), 17.iv.2007, 7 L; Ráztoka brook at Rusava, 370 m (6672), 16.v.2007, 2 đิđ I 1 qI 1 L, all J. Bojková \& S. Zahrádková leg., J. Bojková det. (JBBC); Ratibořka brook above Hošt’álková, 390 m (6673), 14.iv.2011, 2 L, J. Bojková \& T. Soldán leg., J. Bojková det. (JBBC); Jezerní potok brook at Velké Karlovice, $560 \mathrm{~m}$ (6675), 17.iv.2007, 2 đ̊I; Vsetínská Bečva river at

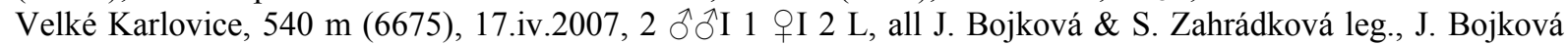
det. (JBBC); Otava river below Sušice, 455 m (6747), 20.iii.1956, 1L, Křelinová leg., J. Bojková det. (NMPC); unnamed brook in Brno-Útěchov, 320 m (6765), 9.v.1948, 1 q I; Svitava river near Babice nad Svitavou, 360 m (6766), 5.iii.1950, 1 đ̊I; all J. Raušer leg., J. Bojková det. (JRBC); Bratřejovka brook above Bratřejov, $400 \mathrm{~m}$ (6773), 14.iv.2011, 1 ठ̊I 19 L, J. Bojková \& T. Soldán leg., J. Bojková det. (JBBC); Ketkovický potok brook

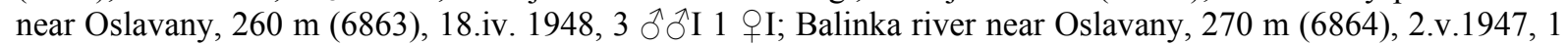

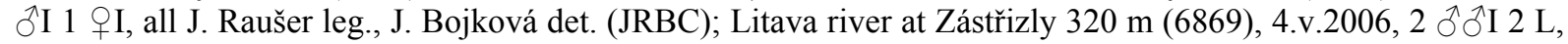
J. Bojková \& S. Zahrádková leg., J. Bojková det. (JBBC); Kudlovický potok brook above Kudlovice, $290 \mathrm{~m}$ (6870), 15.iv.2011, 20 L, J. Bojková \& T. Soldán leg., J. Bojková det. (JBBC); Olšava river above Pitín, 410 m (6973), 23.iv.2008, 1 L, J. Bojková \& S. Zahrádková leg., J. Bojková det. (JBBC); Klenský potok brook near Buškův hamr, 450 m (7153), 2.iii.1999, 7 L, J. Špaček leg. et det. (JSHKC); unnamed springbrook near Mašovice, 360 m (7161), 17.v.1948, 1 †I, J. Raušer leg., J. Bojková det. (JRBC); Klanečnice brook at Květná, 350 m (7172), 14.iv.2011, 2 §ోI 47 L; Malše river in Kaplice, 530 m (7252), 1.iv.2007, 1 L, all J. Bojková \& T. Soldán leg., J. Bojková det. (JBBC).

Distribution and habitat: European species, missing only in the Eastern Plains and southern part of Balkans. In the Czech Republic, it occurs mainly in medium-sized streams at lower altitudes, usually downstream from the habitats of $B$. seticornis.

Remarks: In the 20th century there were only several records of B. risi in Bohemia. In extensive faunistic research of Bohemian Plecoptera in 1955-1960, B. risi was found only at seven of 465 localities of running water habitat (Křelinová 1962). Recently, it was found at 19 of 115 running water localities repeatedly investigated in Bohemia and at 34 other localities. 
The species was more common in Moravia in the past; nowadays it is widespread also in Bohemia. It is a very frequent species especially at submontane and colline habitats, not requiring any species protection status (cf. Soldán et al. 1998, Soldán 2004, Helešic et al. 2005).

\section{Brachyptera seticornis (KLAPÁLEK, 1902)}

(Fig. 4)

Published records: one record from the České Švýcarsko National Park (Bojková et al. 2010); very common in the Jizerské hory Mts. (Preisler \& Špaček 2001), Krkonoše Mts. (Klapálek 1905, Winkler 1977, Špaček 1999, 2001), Rychlebské hory Mts. (Obrdlík 1978), Šumava Mts. (Klapálek 1905, Šámal 1920, Winkler 1956, 1963, Růžičková \& Kotrbová 2000, Soldán et al. 2001, Jezberová 2003); two records from the Českomoravská vrchovina Mts. (Klapálek 1905, 1903, Raušer 1957, unpublished protocols); very common in the Hrubý Jeseník Mts. (Raušer 1955, unpublished protocols, Obrdlík 1981, Tuša 1987, 2001, Soldán et al. 1998), Moravskoslezské Beskydy Mts. (Hrabě et al. 1954, 1958, Straškraba et al. 1954, Raušer 1956, 1958 unpublished protocols, Švec 1960, Soldán et al. 1998, Tuša 2001); two records from the Drahanská vrchovina Mts. (Tuša 2001); one record from the Zábřrežská vrchovina Mts. (Tuša 2001); one record from the Hostýnské vrchy Mts. (Raušer 1958, unpublished protocols), infrequent in the Moravský Kras (Adámek \& Raušer 1977) and Bílé Karpaty Mts. (Bojková et al. 2011).

New records include further regions in South Bohemia - Šumava Mountains foothills, Novohradské hory Mts.; West Bohemia - Slavkovský les Mts., Doupovské hory Mts., Krušné hory Mts.; North Bohemia - Javoří hory Mts., Broumov environs, Orlické hory Mts., Kralický Sněžník Mts.; East Bohemia - Železné hory Mts; and in Moravia - Nízký Jeseník Mts. and Drahanská vrchovina Mts.

Distribution and habitat: Central and South European species. B. seticornis is the most common and abundant species of the family Taeniopterygidae in the Czech Republic. It occurs frequently in clean streams in all mountains above approx. $450 \mathrm{~m}$ a.s.1. Larvae are often topotypic with those of $B$. risi in submontane zone.

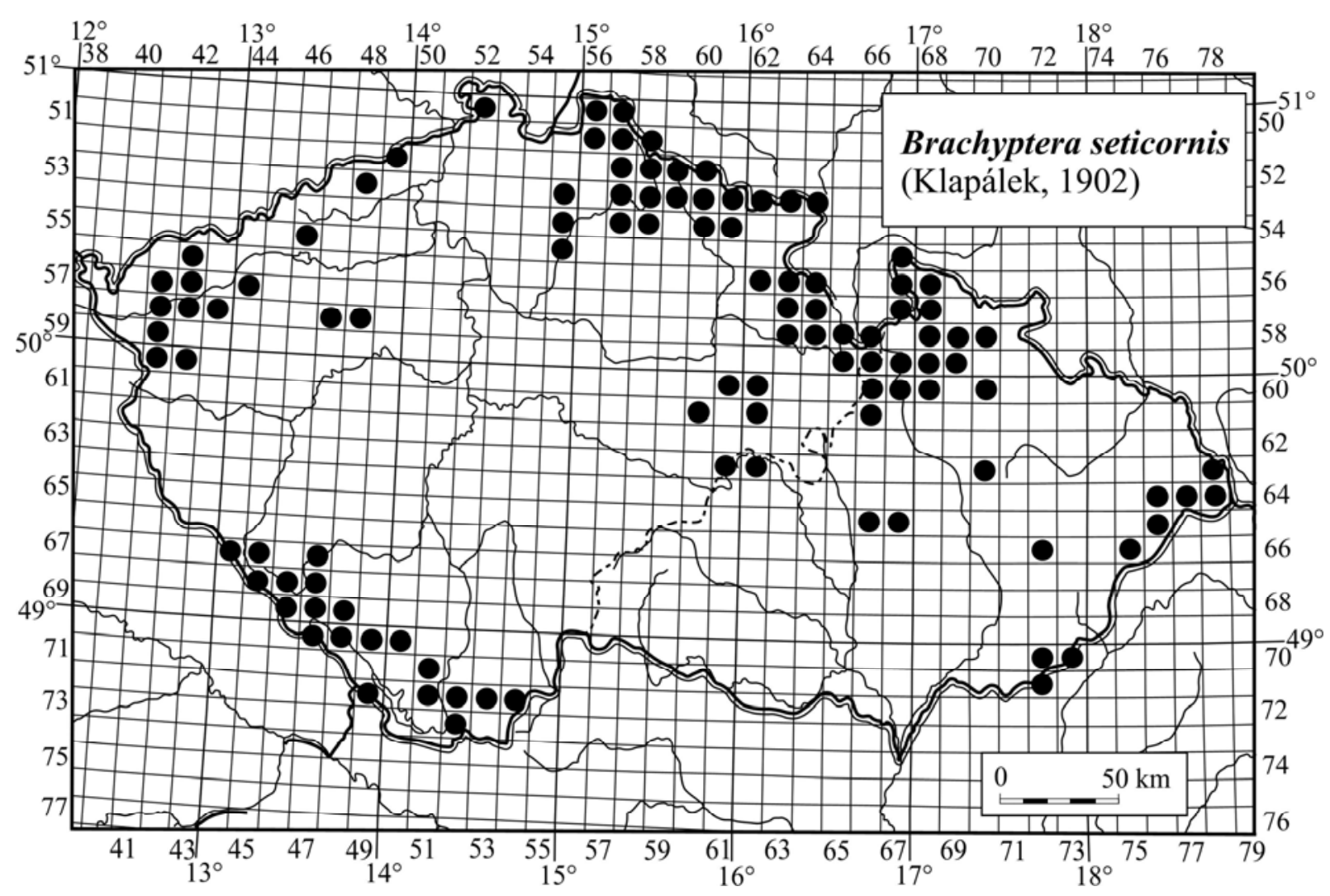

Fig. 4: Distribution of Brachyptera seticornis. All known records are indicated by black circles. 
Remarks: One of the most common species of stoneflies, undoubtedly most frequent species at numerous montane localities, reaching even a considerable quantitative presentation within the whole benthos at riffles. Naturally, the species thus does not require any species protection status (cf. Soldán et al. 1998, Soldán 2004, Helešic et al. 2005).

\section{Brachyptera starmachi SowA, 1966}

(Fig. 5)

Published records: Records by Křelinová and Raušer (Svatopetrský potok brook, springs in Divoký Důl, and Jizera river in Velká Jizerská louka) listed below were formally designated the first records in the Czech Republic by Bojková (2009). Krkonoše Mts. (Špaček 2001, sub B. trifasciata).

New records: Smědá river above Bílý Potok, 570 m (5157), 24.x.1957, 12 L, E. Křelinová leg., J. Bojková det. (NMPC); Velký Kamenický potok brook above Kristiánov, 790 m (5157), 28.ii.2008, 2 웅, J. Bojková leg. et det. (JBBC); Jizera river at Velká Jizerská Louka, 830 m (5158), 7.v.1959, 2 우 ㅇ, E. Křelinová leg., J. Bojková det. (NMPC); Jizerka river below Mísečky, 790 m (5259), 19.xi.2010, 11 L, J. Bojková \& T. Soldán leg., J. Bojková det. (JBBC); Kozelský potok brook at Skelné Hutě, 680 m (5259), 23.x.1963, 9 L, E. Křelinová leg., J. Bojková det. (NMPC); Labe river at Špindlerův Mlýn, 780 m (5259), 8.iii.2008, 8 L, J. Špaček leg. et det. (JSHKC); Svatopetrský potok brook at Svatý Petr, 905 m (5259), 3.iv.1962, 5 đ̊đI, 3 우 II, E. Křelinová leg., J. Bojková det. (NMPC); Modrý potok brook above Pec pod Sněžkou, 890 m (5260), 6.xii.1997, 2 L, J. Špaček leg. et det. (JSHKC), 19.xi.2010, 18 L, J. Bojková \& T. Soldán leg., J. Bojková det. (JBBC); Úpa river above Pec pod Sněžkou, $980 \mathrm{~m}$ (5260), 19.xi.2010, 17 L; Zelený potok brook at Pec pod Sněžkou, $900 \mathrm{~m}$ (5260), 19.xi.2010, 5 L; Jizerka river at Vítkovice, $650 \mathrm{~m}$ (5359), 19.xi.2010, 2 L; Olešenka river at Klárovka at Olešnice v Orlických horách, 610 m (5663), 19.xi.2010, 16 L, all J. Bojková \& T. Soldán leg., J. Bojková det. (JBBC); Bělá river near Šerlišský mlýn at Deštné v Orlických horách, 800 m (5664), 17.xi.1959, 1 đ̂L, 2 우아, E. Křelinová leg., J. Bojková det. (NMPC), 20.xi.2010, 37 L, J. Bojková \& T. Soldán leg., J. Bojková det. (JBBC); Zdobnice river above Zdobnice, 680 m (5764), 21.x.1958, 1 đL 3 3 우 L, E. Křelinová leg., J. Bojková det. (NMPC); Stř́brný potok brook above Nýznerov, $800 \mathrm{~m}$ (5768), 22.xii.2006, 4 L, J. Špaček leg. et det. (JSHKC); Morava river below Horni Hedeč - Pod Mlýnem, 550 m (5966), 22.xii.2006, 1 L, J. Špaček leg. et det. (JSHKC); Bílá Opava river above Karlova Studánka, 820 m (5969), 21. xi. 2010, 15 Lv; Bílá Opava river above Ludvíkov, 690 m (5969), 21.xi.2010, 1 L, all J. Bojková \& T. Soldán leg., J. Bojková det. (JBBC); Podolský

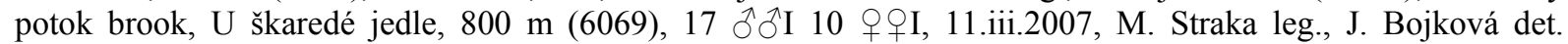
(JBBC); unnamed springs at Divoký Důl, 1330 m (5969), 4.v.1950, 2 우I, J. Raušer leg., J. Bojková det. (JRBC); Jezerní potok brook at Gruberg, $890 \mathrm{~m}$ (6846), 24.x.1956, $2 \delta^{\lambda}{ }^{\lambda} \mathrm{L}$; Křemelná river at Frauenthal, $820 \mathrm{~m}$ (6846), 24.x.1956, 9 L, all E. Křelinová leg., J. Bojková det. (NMPC); Prášilský potok brook at Prášily, $820 \mathrm{~m}$

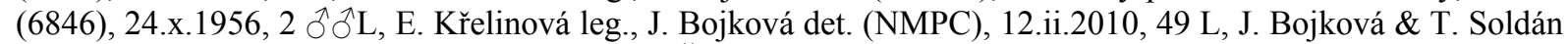
leg., J. Bojková det. (JBBC); Vydra river above Čeňkova Pila, 700 m (6946), 23.x.1956, 1 ठㄴ L, E. Křelinová leg., J. Bojková det. (NMPC); Zhưrský potok brook near Turnerova chata, $840 \mathrm{~m}$ (6947), 29.xi.1956, $2 \delta^{\lambda} \delta^{\lambda} \mathrm{L}, \mathrm{E}$. Křelinová leg., J. Bojková det. (NMPC).

Distribution and habitat: B. starmachi was originally described and known only from the Carpathians (e.g. Sowa 1966, Kis 1972, 1974, Raušer 1977), later it was recorded from the Bavarian Forest (Weinzierl 1999) and the Austrian Alps (Graf \&Weinzierl 2003). In the Czech Republic, it was found in the highest Hercynian mountains. It has not been known in the Carpathians, in the Bílé Karpaty Mts. (Bojková et al. 2011) and Moravskoslezské Beskydy Mts. (J. Kroča, pers. comm.). Larvae were collected predominantly in hypocrenal and epirhithral streams at altitudes between 550 and $1330 \mathrm{~m}$ a.s.1.; most records were above $700 \mathrm{~m}$ a.s.l. The species is locally very abundant.

Remarks: Both larvae and imagines of B. starmachi were often misidentified before its description in 1966. E. Křelinová identified imagines as B. braueri (see above paragraph concerning $B$. braueri) and larvae collected in autumn as $B$. seticornis. There is also possible confusion with $B$. monilicornis recorded in the Hrubý Jeseník Mts. by Losos \& Marvan (1957) as B. starmachi described later is widespread in the Hrubý Jeseník Mts. as well. The species was overlooked for a long time due to its winter life cycle. Imagines and last instars larvae were observed from the end of February till the beginning of March; females were collected even at the beginning of May at the highest altitudes. 


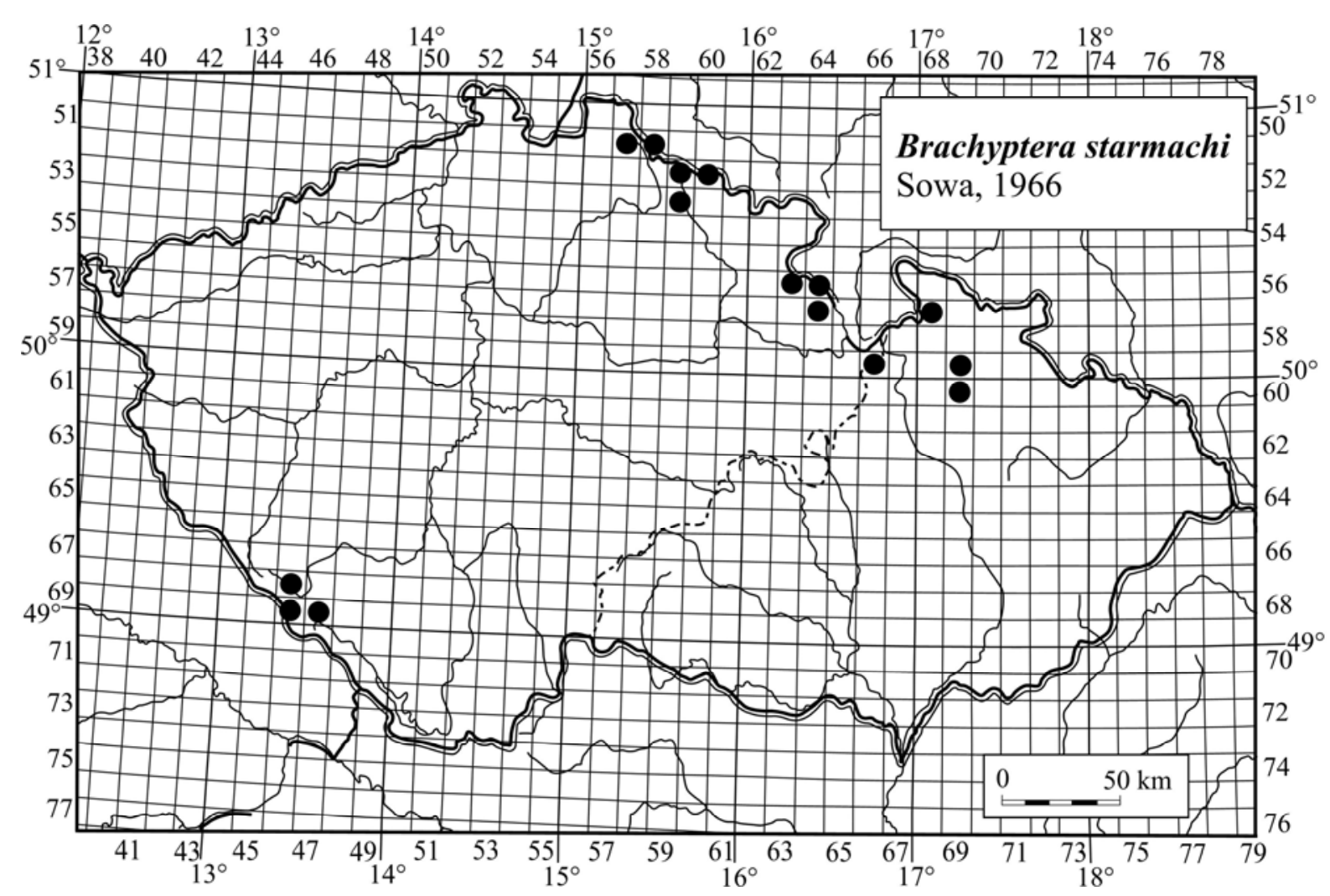

Fig. 5: Distribution of Brachyptera starmachi. Grey circles indicate published records, black circles new records.

\section{Brachyptera trifasciata (PICTET, 1832)}

The species was recorded by Špaček (2001) from the Krkonoše Mts. (exact locality not given). The material was re-identified as B. starmachi. Růžičková et al. (2004) mentioned the species in the Vydra river and Křemelná river basins in the Šumava Mts. during the investigation of benthic larvae assemblages in 1994-1999, but it was not collected again in 2002-2003. Also this record is very likely a misidentification; the larvae are known primarily from large submontane rivers and/or rivers flowing from mountains (cf. Graf \& Hutter 2003). For example, recent findings come from the Alpenrhein river between 600 and $450 \mathrm{~m}$ a.s.1. where the river width is approximately $100 \mathrm{~m}$. No more records of $B$. trifasciata have been published from the Czech Republic, although according to Raušer (1977) it certainly occurred in Bohemia. We failed to trace any data on which the author based his assumption. Possible occurrence of this species within the Czech Republic should be taken with caution. According to Graf \& Hutter (2003) the species was frequently confused with other species and reliable records come from Italy, Switzerland, France, Austria, Germany, Poland, Hungary, Bosnia and Herzegovina, and Turkey. According to Graf \& Hutter (2003) B. trifasciata occurred abundantly in Alpine rivers, but was entirely missing during most of the second half of the last century. It was recently again found at few sites and in low numbers. Since the species has never been found in the Czech Republic, its protection status (regionally extinct) seems to be confusing at this moment (cf. Helešic et al. 2005) and possible occurrence of this species in the Czech Republic should be taken with caution.

\section{Taeniopteryx araneoides KLAPÁLEK, 1902}

$T$. araneoides is now considered extinct in the whole area of its historical distribution, because it has not been collected in the past 100 years (Fochetti \& Tierno de Figueroa 2006). It was a species of large lowland rivers found in Central Europe, in Germany, Hungary and 
Ukraine (Klapálek 1902, 1909, Aubert 1966). Other data on the distribution in the Krasnojar region in Russia should be considered with caution until revision of material collected (Zhiltzova 2003). However provided with the question mark meaning doubtful and/or supposed occurrence in Bohemia (?B), the species has been included in the Raušer's checklist (1997). We failed to trace any data on which the author based this assumption. The only record on possible (but doubtful) occurrence was published by Landa et al. (1997). When checking Klapálek's Ephemeroptera collection together with Prof. Landa in the National Museum in Praha in the late 1970's one of us (T. Soldán) noticed a heavily damaged pinned male specimen in the Plecoptera collection. Posessing only head, part of thorax with right wing base (wing articulation) and anterior part abdomen the specimen was labelled (obviously with Klapálek's hand) "Nephelopteryx araneoides, Praha, Cís. [= Císařská] Louka [= Medow, an island in the Vltava river in Praha]". However, the specimen has never been investigated in detail and has not been definitively found in the Klapálek's collection at present (J. Bojková). The nearest (historical) localities of $T$. araneoides occurrence are the Danube in Budapest (Klapálek 1902) and the Elbe in Dresden (Klapálek 1909). Since this author never mentioned the species from the Czech Republic and there are not any more data of the species occurrence we believe that this matter represents a confusion concerning collection pinned material. The confusion might concern related species, at that time common Brachyptera braueri or Taeniopteryx nebulosa that occurred at the same locality, Císarrská louka (cf. Klapálek 1905). Consequently, the species status "regionally extinct" in the Czech Republic (Helešic et al. 2005) should be considered confusing.

\section{Taeniopteryx auberti KIS ET SowA, 1964}

(Fig. 6)

Published records: Stř́brný potok brook at Nýznerov in the Rychlebské hory Mts. (Obrdlík 1978) and Šumný potok brook above Bělá pod Pradědem in the Hrubý Jeseník Mts. (Obrdlík 1981).

New record: Podolský potok brook, U škaredé jedle, 800 m (6069), 3 §̊đI, 11.iii.2007, M. Straka leg. et det. (JBBC).

Distribution and habitat: Species known from Central Europe, the Carpathians and Balkans. It is widespread (but usually not abundant) especially in the Carpathians, where it inhabits fast flowing montane and submontane streams (e.g. Kis \& Sowa 1964, Kis 1974, Krno 2000, Fiałkowski \& Kittel 2002). In the Czech Republic, it was recorded only from the Hrubý Jeseník Mts. and Rychlebské hory Mts.

Remarks: T. auberti was not included in the red-list of stoneflies by Helešic et al. (2005). Within the Czech Republic the species shows a relict distribution, its area is restricted to two mountain ranges in north Moravia only. Localities known at present are not numerous either in these mountains. Although montane and submontane streams are not subjected to environmental deterioration pressure, the species, like the other representatives of the genus, is sensitive to anthropogenic impacts. We suggest to classify $T$. auberti as endangered species and to add the species to the red-list. We believe that this species, owing to much more restricted area and evidently lower number of actual localities, is more threatened than vulnerable $T$. hubaulti.

\section{Taeniopteryx cf. auberti KIS ET SoWA, 1964}

(Fig. 6)

New records: Kunčický potok brook below Medvědí bouda, 730 m (5767), 29.ix.2007, 1 L; Vidnávka brook near Na Pomezí, 630 m (5768), 22.xii.2006, 2 L; Morava River above Horní Morava, 700 m (5866), 30.ix.2007, 1 L; Morava River below Horní Hedeč - Pod Mlýnem, 550 m (5966), 22.xii.2006, 1 L; Krupá river above confluence with Morava river, Hanušovice, 440 m (5967), 30.ix.2007, 1 L; Morava at Vlaské, 390 m (5967), 22.xii.2006, 8 L, all J. Špaček leg. et det. (JSHKC). 
Remarks: The above cited material includes only larvae which could not be identified with certainty. The identification of larvae, especially small instars, is ambiguous and should be validated by imagines.

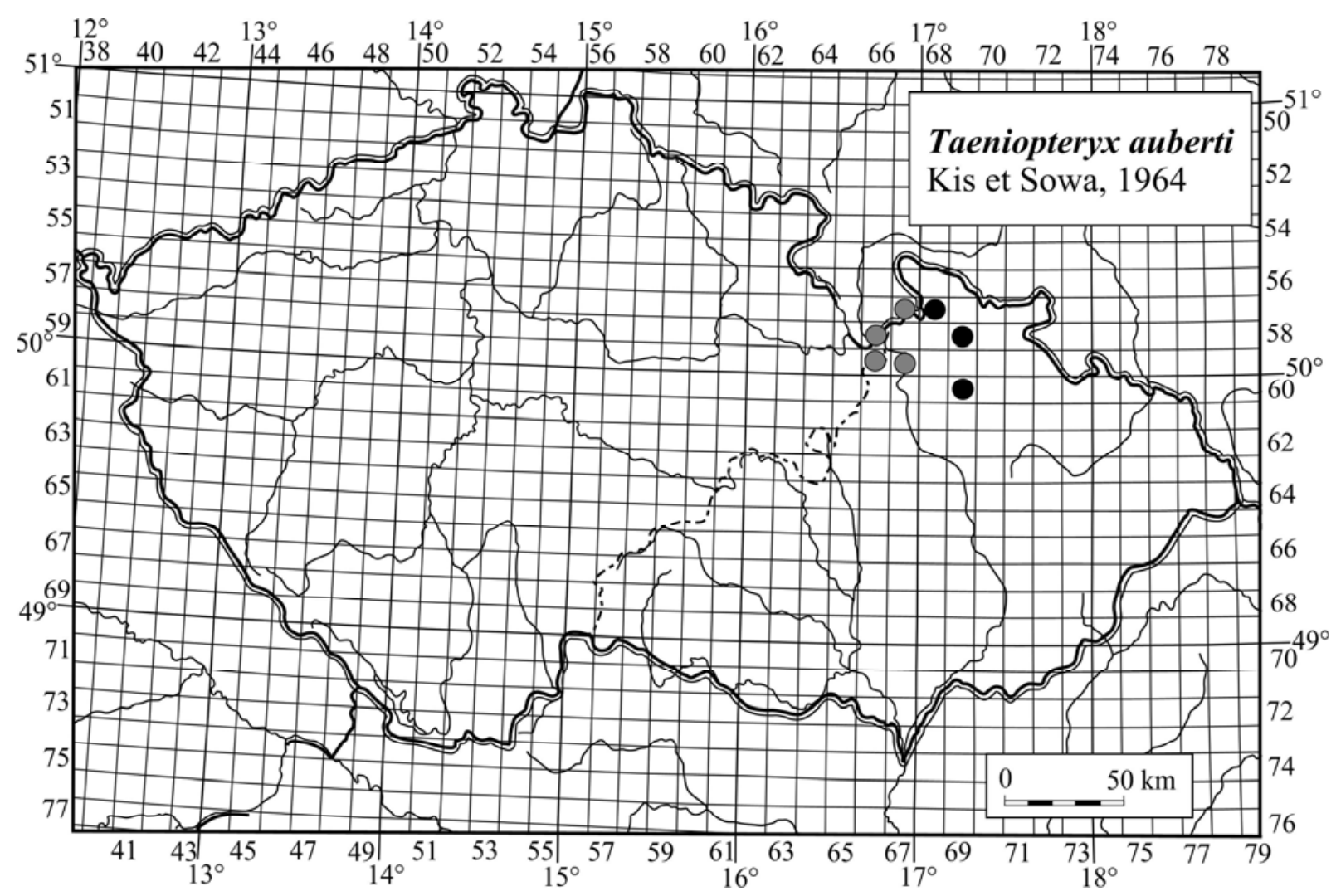

Fig. 6: Distribution of Taeniopteryx auberti. Black circles indicate records of imagines, grey circles records of larvae (T. cf. auberti).

\section{Taeniopteryx hubaulti AUBERT, 1946}

(Fig. 7)

Published records: Křemelná river near Frauenthal, Křemelná river near Čeňkova Pila, and Hradský potok brook near Srní in the Šumava Mts. (Nováková 1958). Nováková (1958) also mentioned Raušer's records from the Morava river near Červený potok and Černá Opava river in Mnichov from the Hrubý Jeseník Mts. by which are also listed in this paper (see below). Jezerní potok brook near Prášily and headwaters of the Úhlava river (Soldán 1996); Křemelná river near Frauenthal (Soldán et al. 2001); Křemelná river and Prášilský potok brook near Vysoké lávky, Slatinný potok brook near Slučí tah (Růžičková et al. 2004b); Podolský potok brook above Janovice in the Hrubý Jeseník Mts. (Losos \& Marvan 1957); Moravice river at Malá Štéhle (Soldán et al. 1998); Černá river in Černé údolí and Keblanský potok brook in Buškův Hamr in the Novohradské hory Mts. (Soldán \& Špaček 2002).

New records: Jizerka river below Mísečky, 790 m (5259), 19.xi.2010, 1 L, J. Bojková \& T. Soldán leg., J. Bojková det. (JBBC); Oharka brook near Libíć, 320 m (5356), 26.x.2004, 9L; Lysečinský potok brook near Dolní Lysečiny, 610 m (5360), 31.x.2008, 1 L, J. Špaček leg. et det. (JSHKC); Malé Labe river at Prosečné, 390 m (5460), 20.x.1958, 2 L, E. Křelinová leg., J. Bojková det. (NMPC); Ličná river near Poříćí u Trutnova, 390 m (5461), 11.ix.2006, 18 L; Javorník brook above Javorník, 360 m (5667), 22.xii.2006, 1 L; Račí potok brook near Račí údolí, 360 m (5667), 22.xii.2006, 9 L; Zdobnice river near Bělá, 490 m (5763), 1 L; Branná river above Branná, 640 m (5868), 19.x.1999, 1 L, all J. Špaček leg. et det. (JSHKC); Černá Opava river at Mnichov, 590 m (5870), 20.x.1955, 1 L; Morava river near Červený potok, 550 m (5966), 22.iv.1955, 1 † I, 1 L, all J. Raušer leg., J. Bojková det. (JRBC); Bílá Opava river above Ludvíkov, 690 m (5969), 21.xi.2010, 1 L, J. Bojková \& T. Soldán leg., J. Bojková det. (JBBC); Moravice river at Malá Št'áhle, 590 m (6070), 18.x.1999, 5 L; Pstružný potok brook at Dolní Chlum, $610 \mathrm{~m}$ (6746), 12.ix.2008, 3 L; Otava river at Červené Dvorce, $490 \mathrm{~m}$ (6747), 16.x.1998, 1 L, all J. Špaček leg. et det. (JSHKC); Otava river at Rabí, 440 m (6747), 9.ii.2011, 1 L, J. Bojková \& T. Soldán leg., J. Bojková det. (JBBC); Arnoštský potok brook above Solná Lhota, 790 m (6948), 5.xi.1959, 6 
L; Polecký potok brook near Slatina, 830 m (7048), 5.xi.1959, 6 L; Teplá Vltava river near Slatina, $830 \mathrm{~m}$ (7048), 5.xi.1959, 2 L all E. Křelinová leg., J. Bojková det. (NMPC); Blanice river near Blažejovice, $770 \mathrm{~m}$ (7049), 15.x.1998, 14 L; Malše river near Dolní Dvořiště, 620 m (7352), 14.x.1999, 16 L, all J. Špaček leg. et det. (JSHKC).

Distribution and habitat: European species missing in Mediterranean, Fennoscandia and British Islands. In the Czech Republic, it occurs mainly in high Hercynian mountains: the Krkonoše Mts., Orlické hory Mts., Rychlebské hory Mts., Hrubý Jeseník Mts., Šumava Mts. and the Novohradské hory Mts. It inhabits cold and clean, fast flowing, medium-sized streams in montane and submontane zones.

Remarks: Helešic et al. (2005) considered the species as vulnerable. Taking into account relatively high number of both historical and new localities of the occurrence and relatively wide distribution within the whole Hercynian system in the Czech Republic, this classification seems to be rather overestimated and the category NT (near threatened) would probably better describe its real status. Moreover, at some localities (e.g., Křemelná river in the Šumava Mts.), larvae are very abundant. The species probably escaped our attention due to a typical winter life cycle (larvae start to appear in late September, imagines fly very early, usually in February and March).

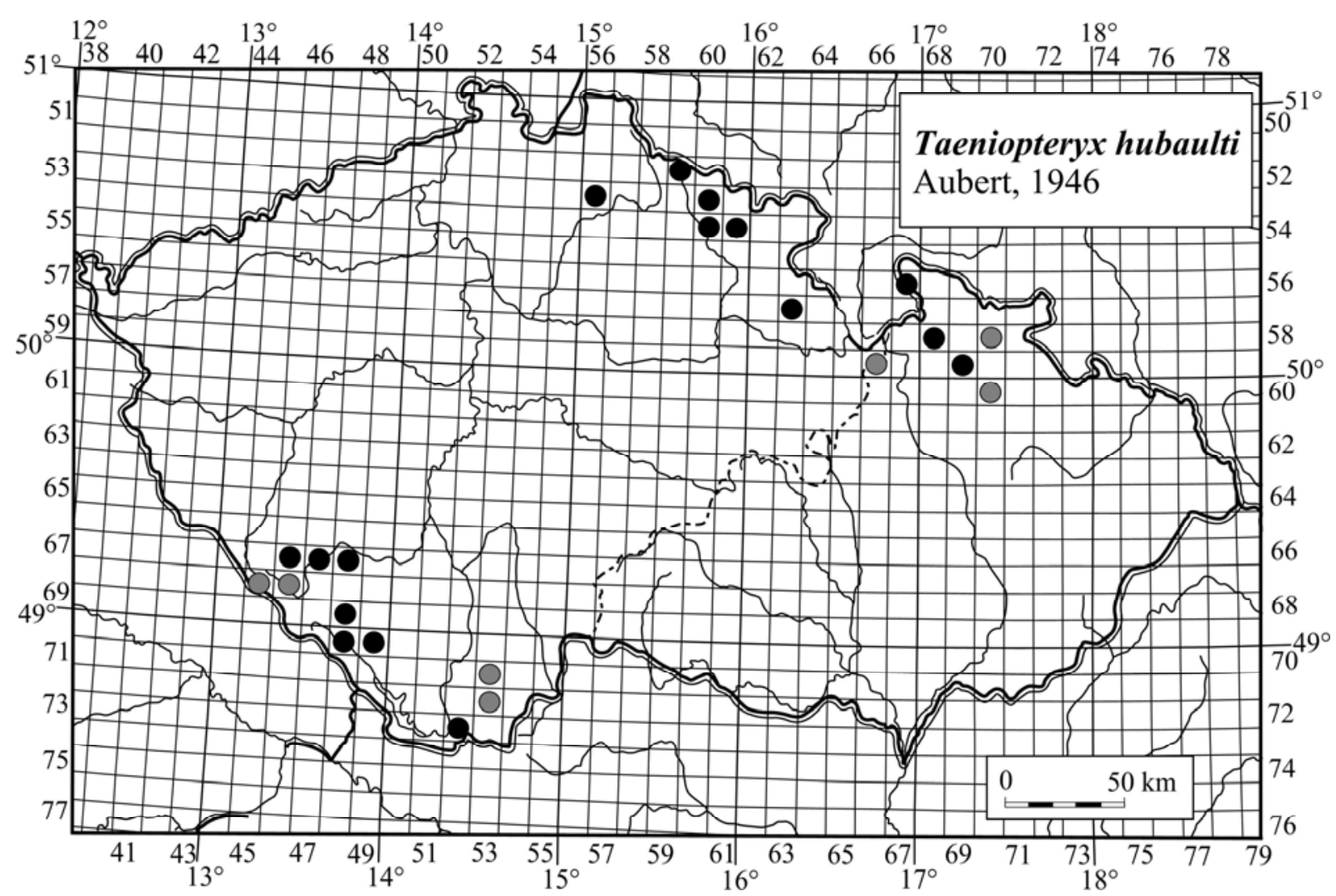

Fig. 7: Distribution of Taeniopteryx hubaulti. Grey circles indicate published records, black circles new records.

\section{Taeniopteryx kuehtreiberi AUBERT, 1950}

Published records: Ostružná river near Velhartice (Soldán 1996)

Distribution and habitat: Central and South European species living at montane rivers (Aubert 1959, Ravizza \& Fochetti 1999). These authors observed T. kuehtreiberi predominantly at altitudes above $1000 \mathrm{~m}$ a.s.1.

Remarks: T. kuehtreiberi was incorporated into fauna of the Czech Republic for the first time by Raušer (1977). He classified its occurrence without any doubt, but we failed to trace 
a concrete source of this opinion. Based on this classification we can speculate that Dr. Raušer has really seen some material. Later, Raušer (1980) mentioned the occurrence of this species in his key stating that the species is distributed in western Bohemia. Its real occurrence in the Czech Republic cannot be excluded. On the other hand, typical localities of its occurrence are not present in Western Bohemia (cf. Aubert 1959, Ravizza \& Fochetti 1999). Unfortunately, Soldán's (1996) larval material has been lost and thus cannot be verified. Confusion with more frequent $T$. hubaulti distributed at the same area cannot be excluded. Helešic et al. (2005) considered the species as regionally extinct. Due to the absence of voucher specimens we do not consider T. kuehtreiberi a part of the Czech fauna.

\section{Taeniopteryx nebulosa (LINNAEUS, 1758)}

\section{(Fig. 8)}

Published records: Vltava river in Praha-Štvanice and at Císařská louka; Zlatá stoka stream in Třeboň; Nežárka river in Veselí nad Lužnicí; Plzeň (Klapálek 1905); Praha-Zátiší (coll. Klapálek); Vltava river near Frymburk (Nowak et al. 1935, Winkler 1963, before establishment of the Lipno Reservoir).

New records: Jizerka river near Jizerka, 870 m (5157), 28.x.2005, 4 L; Safírový potok brook above Jizerka, 870 m (5157), 28.x.2005, 2 L, all J. Špaček leg. et det. (JSHKC); Jizera river at Kořenov, 570 m (5258), 26.ix.1957, 1 L, E. Křelinová leg., J. Bojková det. (NMPC); Oharka brook near Libíč, 320 m (5356), 26.x.2004, 4 L, J. Špaček leg. et det. (JSHKC); Žehrovka river at Březina, 240 m (5456), 25.x.1957, 2 L; Ohře river in Kadaň, 290 m (5645), 23.xi.1955, 1 L; Ohře river in Postoloprty, 180 m (5648), 21.x.1957, 3 L, all E. Křelinová leg., J. Bojková det. (NMPC); Hamerský potok brook near Planá, 480 m (6142), 13.x.1999, 1 L; Mže river above Milíkov, 400 m (6243), 13.x.1999, 2L, 29.xii.2006, 4 L, all J. Špaček leg. et det. (JSHKC); Blanice river at Radonice, 270 m (6255), 25.x.2008, 3 L, M. Straka leg. et det. (JBBC); Svratka river near Cikháj, $2900 \mathrm{~m}$ downstream a spring, 670 m (6361), 1 L, 22.ix.1960, 1 L, 25.x.1960, E. Křelinová leg., J. Bojková det. (NMPC),

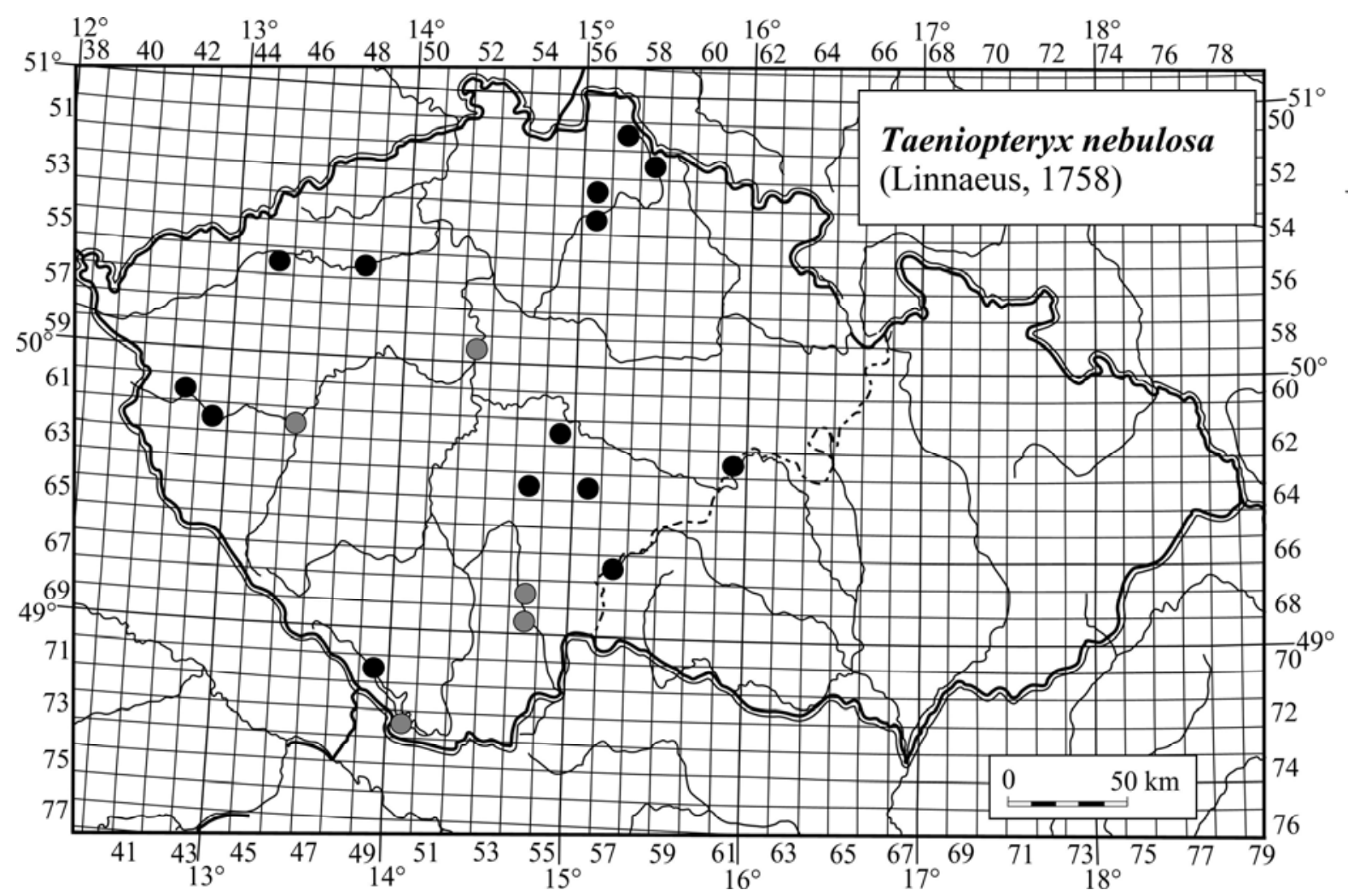

Fig. 8: Distribution of Taeniopteryx nebulosa. Grey circles indicate published records, black circles new records.

19.ix.1957 5 L, 16.x.1957 2 L, 13.xi.1957 4 L; 6.iii.1958, 1 E, 1 đII (damaged material), J. Raušer leg., J. Bojková det. (JRBC); Trnava river near Hladov, 550 m (6454), 26.x.1999, 14 L; Martinický potok brook near Borek 470 m (6456), 25.x.1999, 3 L, all J. Špaček leg. et det. (JSHKC); Žirovnice river near Cholunná, 550 m 
(6757), 17.x.1957, 2 L, J. Raušer leg., J. Bojková det. (JRBS); Vltava river near Pěkná, 730 m (7149), 4.xi.1959, 9 L, E. Křelinová leg., J. Bojková det. (NMPC); 15.x.1998, 20 L, J. Špaček leg. et det. (JSHKC); 10.xi.2008, 6 L, J. Bojková \& T. Soldán leg., J. Bojková det. (JBBC).

Distribution and habitat: Transpalaearctic species according to Zhiltzova (1997) with the occurrence in south Siberia, Amur basin, Primorye and Sachalin. Evenly distributed within the whole Europe including the British Islands, except for some regions in west Balkan. It occurs in hyporhitral-metapotamal streams mainly in coline and lowland zones, rarely in submontane and montane zones.

Remarks: Till the late 1950's T. nebulosa represented a rare but rather equally distributed species within the Czech Republic at a relatively high altitudinal range of about 180-730 m a.s.l. evidently inhabiting a large scale of habitats from lowland rivers (Vltava river in Prague) up to submontane rivers (Jizera river at Kořenov, Svratka river at Cikháj). Recent records are from medium-size streams in colline and montane zones. It was not found in any potamal locality. It was not found at localities of its historical occurrence, except the Vltava river above Lipno reservoir. Similarly to many sensitive potamal species, it dramatically decreased due to blanket degradation of habitats. Helešic et al. (2005) found it to be vulnerable in the Czech Republic. Due to its evident disappearance from the vast majority of known historical localities it should be classified rather as endangered (cf. also Soldán 2004), taking into account to general rules depicted for species protection in the Czech Republic by Farkač et al. (2005).

\section{Oemopteryx loewii ALBARDA, 1889}

Oe. loewii is now considered extinct in the whole species distribution area, because it has not been collected during the past 100 years (Fochetti \& Tierno de Figueroa 2006). It occurred in large European streams (the Rhine between Bonn and Arnhem and the Danube betweem Budapest and Regensburg) (Klapálek 1909, Zwick 1992, 2004). A few females are preserved in museums, but no single male. The larva has never been found. Data on the occurrence of Oemopteryx loewii in the Czech Republic are based solely on misidentification; this species is definively not documented from this area. According to Bojková (2009), two females of Oe. loewii collected in the Střela River in Western Bohemia (Nováková 1958, Křelinová 1962) and deposited in Křelinová's collection in the National Museum in Prague were evidently misidentified, confused with females belonging to different genera of Taeniopterygidae. There is no other record of the species in the Czech Republic. Data in the Raušer's check-list (1977) and in the Red List by Helešic et al. (2005) were based on this incorrect record.

\section{Rhabdiopteryx acuminata KLAPÁLEK, 1905}

The only literature records on the occurrence of this species in the Czech Republic are those by Soldán (2004), who mentioned two larvae collected in the Křemelná river near Antygl in the Šumava Mts., and Soldán et al. (1998) who recorded it in the Černá Opava river at Mnichov. The first record (Soldán 2004, p. 200) is a purely typing error, name confusion with the species $R h$. neclecta which is correctly presented in preceding table in the same contribution (Soldán 2004, p. 198). The second record is based on the larval material, which is not available at the moment. Rh. acuminata was not re-collected in the above mentioned locality at present. Its occurrence in the Czech Republic cannot be excluded, since this region is situated within the area of this species. It occurs in British Islands, Central and North Europe (Finland, Latvia, and Lithuania) with an eastern area extension to the Smolensk and Novgorod regions and Komi Autonomous Republic of Russia (Zhiltzova 1997, 2003). It was recorded in the vicinity of the borders of the Czech Republic, e.g. upper part of Visla (Vistula) river and Orava river basins (Raušer 1980, Fiałkowski \& Kittel 2002, Krno 2004). 


\section{Rhabdiopteryx neglecta (ALBARDA, 1889)}

The occurrence of Rh. neglecta in the Czech Republic is questionable. It is mentioned from this area for the first time by Raušer $(1959$, p. 170) in his key to imagines of Plecoptera of Czechoslovakia. Although no concrete locality is presented, he wrote: "... a species known from the Odra (Oder) river in March and April, probably also in our country". However, later Raušer (1977) evidently abandoned the idea on the occurrence of Rh. neglecta in the Czech Republic, citing the species neither from Bohemia nor from Moravia in his check list. Soldán et al. (1998, p. 102) also mentioned the finding of the species in the Odra river basin, but locality was not provided. Other records of Rh. neglecta are from the Šumava (Bohemian Forest) Mts. Růžičková \& Kotrbová (2000) found it in the Roklanský potok brook in 1997. Růžičková et al. (2004a,b) mentioned the occurrence in the Vydra river and Křemelná river basins in the 1990s, which was not observed at the same localities in 2002-2003. Soldán's record (2004) is from the same area, from the Křemelná river near Antygl (as Rh. acuminata, see the above respective paragraph). All these findings are based on larvae, sometimes most probably on younger ones, the determination of which is complicated, sometimes even not reliable. Consequently, there is an assumption that this identification based on larvae is not correct and the larvae might belong even to related genus Brachyptera. Unfortunately, all the material is not available. On the other hand, the occurrence of $R h$. neglecta in the Czech Republic seems to be probable owing to relatively large area of $R h$. neglecta representing a Central and South European species (Ravizza \& Fochetti 1999, Vinçon \& Murányi 2009). The species is distributed also in the vicinity of the Czech Republic borders, e.g. in Austria and Bavaria. Due to the absence of voucher specimens we do not consider Rh. neglecta a part of the Czech fauna.

\section{Other species of the genus Rhabdiopteryx}

Concerning other representatives of the genus Rhabdiopteryx, the occurrence of some additional species in the Czech Republic is very likely. It can concern $R h$. navicula Theischinger, 1974 living in the area very close to southern border of Bohemia (Mühlviertel in Austria, further localities in the Inn river basin) at the habitats of middle-sized brooks with prevailing riffles, usually together with Brachyptera risi and B. seticornis (cf. Theischinger 1974). Thanks to an excellent revision of the Rhabdiopteryx neglecta species-group in Europe by Vinçon \& Murányi (2009), we can suppose the occurrence of some further species of the genus. While the distribution $R h$. alpina Kühtreiber, 1934 (originally supposed to occur also in the Carpathians) is now restricted solely to the Alps, its occurrence in the Czech Republic does not seem to be likely. We can rather expect findings of recently established $R h$. harperi Vinçon et Murányi, 2009, which has a bow-like area in both the Alps and Carpathians (so far known from southeastern Poland, Ukraine and Romania). Presumable area (Vinçon \& Murányi 2009, p. 215, Fig. 29) comprises a part of south Bohemia (the Šumava Mts.) and Moravian and Slovakian Carpathian mountains.

\section{Conclusion}

Altogether 14 species were previously mentioned from the Czech Republic. Eleven of them were listed in the only existing check-list of Plecoptera of the Czech Republic (Raušer 1977), the others were recorded later.

In total eight species were found to occur with certainty in the Czech Republic. Two species, namely Brachyptera seticornis and B. risi, are widespread and abundant. The latter exhibited some expansion in Bohemia at present. B. starmachi, which was earlier often confused with $B$. seticornis or misidentified as $B$. braueri, was recorded numerously in all high Hercynian mountains. It was abundant in clear montane streams, thus it was classified 
near threatened. Another species, Taeniopteryx hubaulti, is known from numerous localities in the Hercynian mountains, although the species was found individually. Due to relatively wide distribution it was classified near threatened. Two species, B. monilicornis and $T$. auberti, were found only in several records in restricted area of the Czech Republic. The former was recently found only in streams at the foothills of the Šmava Mts.; the latter was found only in montane and submontane streams in the Hrubý Jeseník Mts. and the Rychlebské hory Mts. Due to their restricted area were classified as endangered. Two species were found to be critically endangered due to considerable decline in the second half of the 20th century. Brachyptera braueri was missing last 40 years; recently, it has been found in several rivers showing some recovery of the original area. Taeniopteryx nebulosa is recently known only from montane or submontane streams; it dramatically decreased in lowland and colline streams which were its typical habitats.

No reliable data have been found as to distribution of Brachyptera trifasciata and Oemopteryx loeii which were misidentified in the past. We found no reliable data or material of Taeniopteryx kuehtreiberi and T. araneoides. The former species was reported from Western Bohemia, the latter was mentioned from the Vltava river in Praha, but original material was lost. The occurrence of these species in the Czech Republic is doubtful and formally cannot be considered being a part of Czech fauna without the voucher material. The occurrence of two species of Rhabdiopteryx (R. acuminata and R. neglecta) reported from the Šumava Mts. and the Odra river basin is doubtful at this moment, because it is based on the problematic determination of larval material. Some species of the genus Rhabdiopteryx can be supposed, but there are no actual distributional data and concrete material available at present.

Acknowledgements: This study was supported by the long-term research plan of Masaryk University (MSM 0021622416) and the research project of the Czech Science Foundation (P505/10/P302).

\section{References}

Adámek Z. (1972): Příspěvek k rozšíření Brachyptera braueri (Klapálek 1900) v ČSSR (Plecopt., Taeniopterygidae).- Zprávy Československé Společnosti entomologické, Praha, 8: 85-88.

Adámek Z. \& Raušer J. (1977): Příspěvek k otázce čistoty vodních toků Moravského krasu na příkladě studia fauny jepic (Ephemeroptera) a pošvatek (Plecoptera).- Speleologický věstník, 8: 7-23.

Aubert J. (1959): Plecoptera. Insecta Helvetica, Fauna 1. Imprimerie La Concorde, Lausanne, 140 pp.

- (1966): Notes sur quelques Plécoptères du Muséum d'Histoire Naturelle de Budapest.- Annales historiconaturales Musei nationalis Hungarici, pars zoologica, 58: 285-288.

Bojková J. (2009): Revision of the stonefly collections (Plecoptera) by E. Křelinová and J. Raušer from the Czech Republic.- Aquat. Insects, 31(suppl. 1): 249-255.

Bojková J., Chvojka P. \& Komzák P. (2011): Stoneflies (Plecoptera) of the Bílé Karpaty Protected Landscape Area and Biosphere Reserve.- Acta Musei Moraviae, Scientiae biologicae (Brno). (in print)

Bojková J., Soldán T., Zahrádková S., Chvojka P. \& Trýzna M. (2010): Ephemeroptera and Plecoptera of the Bohemian Switzerland National Park, Czech Republic: species diversity and taxocenoses of sandstone watercourses.- Lauterbornia, 70: 91-110.

Bojková J. \& Špaček J. (2006): New and interesting records of Plecoptera (Insecta) from the Czech Republic.- Acta Musei Moraviae, Scientiae Biologicae (Brno), 91: 1-6.

Brettfeld R. \& Bellstedt R. (2000): Wiederfund der Steinfliege Brachyptera braueri (Klapálek, 1900) nach fast hundert Jahren in Thüringen (Insecta: Plecoptera).- Abhandlungen und Berichte des Museums der Natur Gotha, 21: 111-112

- (2003): Die Veirbreitung der flussbewohnenden Steinfliege Brachyptera braueri (Klapálek, 1900) in Werra and Saale, zugleich ein Beitrag zur Situation der Flussinsekten in Thüringen.- Veröffentlichungen Naturhistorisches Museum Schleusingen, 18: 69-78.

Dorn A. \& Weinzierl A. (2003): Ein Beitrag zur Kenntnis der Eintags- und Steinfliegen-Fauna (Ephemeroptera; Plecoptera) der niederbayerischen Isar-- Lauterbornia, 47: 21-26.

Ehrendorfer F. \& Hamann U. (1965): Vorschläge zu einer floristischen Kartierung von Mitteleuropa.Berichte der Deutschen Botanischen Gesellschaft, 78: 35-50.

Farkač J., Král D. \& Škorpík M. (eds) (2005): Červený seznam ohrožených druhů České republiky. Bezobratlí. Agentura ochrany přírody a krajiny České republiky, Praha, 760 pp. 
Fiałkowski W. \& Kittel W. (2002): Widelnice Plecoptera. Katalog fauny Polski część XVI, zeszyt 3. Muzeum i Instytut zoologii PAN, Warszawa, 72 pp.

Fochetti R. \& Tierno de Figueroa J.M. (2006): Notes on diversity and conservation of the European fauna of Plecoptera.- J. Nat. Hist., 40: 2361-2369.

Graf W. (1999). Check-Liste der Steinfliegen (Insecta: Plecoptera) Österreichs.- Lauterbornia, 37: 35-46.

Graf W., Grasser U. \& Wenzierl A. 2002: Plecoptera. - Part III, pp. 1-17. In Moog O. (ed.): Fauna Aquatica Austriaca, Edition 2002. Wasserwirtschaftskataster, Bundesministerium für Land- und Forstwirtschaft, Umwelt und Wassewitschaft, Wien.

Graf W. \& Hutter G. (2003): Recent findings of the endangered stonefly Brachyptera trifasciata (PICTET, 1832) in Austria with a description of its habitat, pp. 305-307. In Gaino E. (ed.): Research update on Ephemeroptera and Plecoptera. University of Perugia, Perugia, $488 \mathrm{pp}$.

Graf W., Lorenz A.W., Tierno de Figueroa J.M., Lucke S., Lopez-Rodriguez M.J. \& Davies C. (2009): Plecoptera. In Schmidt-Kloiber A. \& Hering D. (eds): Distribution and ecological preferences of European freshwater organisms, volume 2. Pensoft Publishers, Sofia, $262 \mathrm{pp}$.

Graf W. \& Wenzierl A. (2003): Distribution of Brachyptera starmachi Sowa, 1966 and Capnia vidua rilensis Raušer, 1962, pp. 309-311. In Gaino E. (ed.): Research update on Ephemeroptera and Plecoptera. University of Perugia, Perugia, 488 pp.

Helešic J., Soldán T. \& Špaček J. (2005): Plecoptera (pošvatky), pp. 128-130. In Farkač J., Král D. \& Škorpík M. (eds): Červený seznam ohrožených druhů České republiky. Bezobratlí. Agentura ochrany prírody a krajiny České republiky, Praha.

Hohmann M. (2004): Nachweise von Brachyptera braueri (Plecoptera, Taeniopterygidae) in der Mulde/Elbe, Sachsen und Sachsen-Anhalt.- Lauterbornia, 50: 75-78.

Hrabě S., Obr S., Kubíček F., Raušer J., Zelinka M. \& Marvan P. (1954): Zpráva o výsledcích hydrobiologických šetření v povodí budoucí přehrady u Šanců. Zoologický ústav Př́rodovědecké fakulta university v Brně a Výzkumný ústav vodohospodářský Praha, laboratoře v Brně, 37 pp.

Hrabě S., Kubíček F., Obr S., Raušer J., Zelinka M. \& Knoz J. (1958): Výsledky hydrobiologických šetření v povodí budoucí údolní nádrže na Morávce. Zoologický ústav Př́rodovědecké fakulta university $\mathrm{v}$ Brně, 29 pp.

Illies J. (1978): Plecoptera, pp. 264-273. In Illies J. (ed.): Limnofauna Europea. Gustav Fischer Verlag, Stuttgart, $532 \mathrm{pp}$

Jezberová M. (2003): Distribution and density of Ephemeroptera and Plecoptera of the Radíkovský brook (Czech Republic) in relation to selected environmental variables, pp. 327-331. In Gaino E. (ed.): Research Update of Ephemeroptera \& Plecoptera. University of Perugia, Perugia, 488 pp.

Kis B. (1972): Genul Brachyptera New. (Plecoptera) in R.S. Romania.- Studia Universitatis Babeş -Bolyai, Series Biologia, 1: 107-111.

- (1974): Plecoptera, Insecta. Fauna Republicii Socialiste România. Vol. 8. Editura Academiei Republicii Socialiste România, Bucuresti, 273 pp.

Kis B. \& Sowa R. (1964): Taeniopteryx auberti n. sp., eine neue Plecopterenart aus den Karpathen.- Bulletin de l'Académie polonaise des sciences Cl. II, 12(8): 343-345.

Klapálek F. (1902): Beitrag zur Kenntnis der Neuropteroiden von Ungarn, Bosnien und Herzegovina.Termes. Füset., 25: 161-180.

- (1903): Zpráva o výzkumu českých Neuropteroid v r. 1902.- Věstník České akademie císaře Františka Josefa pro vědy, slovesnost a umění, 12(5): 257-264.

- (1905): Conspectus Plecopterorum Bohemiae.- Časopis České Společnosti entomologické, II(1): 29-31.

- (1906): Klíč evropských druhů čeledi Taeniopterygidae.- Časopis České Společnosti entomologické, III(4): 91-96.

- (1909): Plecoptera, Steinfliegen, pp. 33-95. In Brauer A. (ed.): Die Süsswasserfauna Deutschlands, Heft 8. Verlag von Gustav Fischer, Jena, 163 pp.

Krno I. (2000): Rozšírenie pošvatiek (Plecoptera) na Slovensku. Správy Slovenskej zoologickej spoločnosti 18: 39-54.

- (2004). Distribution and phylogenetic relationships of the genus Rhabdiopteryx (Plecoptera, Taeniopterygidae) in Slovakia.- Biologia, Bratislava, 59(2): 181-190.

Křelinová E. (1962): K poznání českých pošvatek (Plecoptera). Studie o bionomii a zoogeografii bentické české zvíreny. [To the knowledge of the Czech stoneflies (Plecoptera). A study of bionomy and zoogeography of the Czech benthic fauna]. (PhD thesis). Institute of Entomology, 1962, Prague, $265 \mathrm{pp}$.

Küttner R., Hohmann M., Plesky B. \& Voigt H. (2008): Zur Verbreitung und Ökologie von Brachyptera braueri (Klapálek, 1900) (Insecta: Plecoptera) in Mitteldeutschland unter Berücksichtigung weiterer Plecoptera-Arten des zeitigen Frühjahrens.- Lauterbornia, 63: 31-50.

Le Doaré J. \& Vinçon G. (2006): Les Plécoptères de France: inventaire des espèces signalées par départements [Plecoptera].- Ephemera [2005], 7(1): 11-43. 
Landa V., Helešic J., Soldán T. \& Zahrádková S. (1997): Stoneflies (Plecoptera) of the River Vltava, Czech Republic: a century of extinction, pp. 288-295. In Landolt P. \& Sartori M. (eds): Ephemeroptera \& Plecoptera: Biology-Ecology-Systematics. Maron+Tinguely \& Lachat SA, CH-Fribourg, 569 pp.

Losos B. \& Marvan P. (1957): Hydrobiologické poměry řeky Moravice a jejích přítoků Podolského a Černého potoka.- Sborník Vysoké školy zemědělské a lesnické v Brně, řada A: spisy Fakulty agronomické a zootechnické, 1: 41-69.

Malicky-Ruzicka H.M. \& Malicky M. (2003): Rückkehr einer bedrohten Steinfliegenart oder Sammelartefakt - Versuch einer Interpretation am Beispiel Brachyptera monilicornis (Insecta: Plecoptera, Taeniopterygidae).- Lauterbornia, 47: 27-32.

Murányi D. (in litt.): A Karpat-medence és a Balkán álkérész (Plecoptera) faunájának taxonómiai problémái, állatföldrajzi vizsgálata. [Taxonomical problems and zoogeographical investigation of the stonefly (Plecoptera) fauna of the Carpathian Basin and the Balkans]. (PhD thesis). Eötvös Loránd Tudományegyetem, Állatrendszertani és Ökológiai Tanszék, Biológia Doktori Iskola, 2008, 149 pp.

Novák I. (1989): Seznam lokalit a jejich kódù pro sít’ové mapování entomofauny Československa.- Zprávy Československé společnosti entomologické při ČSAV, 25: 3-84.

Nováková E. (1958): Příspěvek k poznání pošvatek Čech (Plecoptera).- Časopis Československé Společnosti entomologické, 55: 174-176.

Nowak W., Sýkora L. \& Dechant E. (1935): Výsledek biologického výzkumu Vltavy mezi Frymburkem a Týnem n. Vlt., pp. 82-106. In Studie o znečištění vody horní Vltavy, České Budějovice.

Obenberger J. (1958): Entomologie IV. Systematická část 3. Nakladatelství Československé akademie věd, Praha, 614 pp.

Obrdlík P. (1978): Rheobentos a jakost vody Stříbrného potoka v Rychlebských horách.- Čas. Slez. Muz. Opava (A), 28: 69-75.

- (1981): K poznání hydrobiologie Borového a Šumného potoka v Hrubém Jeseníku z hlediska čistoty vody.Čas. Slez. Muz. Opava (A), 30: 89-95.

Preisler J. \& Špaček J. (2001): Pošvatky (Plecoptera) a střechatky (Megaloptera) Jizerských hor.- Sborník Severočeského Muzea - Př́rodní vědy, Liberec, 22: 109-114.

Pruner L. \& Míka P. (1996): Seznam obcí a jejich částí v České republice s čísly mapových polí pro sít’ové mapování fauny.- Klapalekiana, 32 (Suppl.): 1-115.

Raušer J. (1959): Ř́d pošvatky - Plecoptera, pp. 169-171. In Kratochvíl J. (ed.): Klíč zvířeny ČSR III. Nakladatelství Československé akademie věd, Praha, 869 pp.

- (1980): Ř́d pošvatky - Plecoptera, pp. 86-132. In Rozkošný R. (ed.): Klíč k určování vodních larev hmyzu. Academia, Praha, $521 \mathrm{pp}$.

- (1977): Plecoptera, pp. 27-29. In Dlabola J. (ed.): Enumeratio insectorum Bohemoslovakiae.- Acta faunistica entomologica Musei nationalis Pragae, Supplementum 4: 1-159.

- (1992): Pošvatky, pp. 66-69. In Škapec L. (ed.): Červená kniha ohrožených a vzácných druhů rostlin a živočichů ČSFR 3, Bezobratlí. Príroda, Bratislava, 155 pp.

Ravizza C. \& Fochetti R. (1999): I Plecotteri Taenyiopterygidae della regione italica (Plecoptera).- Mem. Soc. entomol. ital., 77: 123-159.

Reusch H. \& Weinzierl A. (1999): Regionalisiete Checkliste der aus Deutschland bekannten Steinfliegenarten (Plecoptera).- Lauterbornia, 37: 87-96.

Růžičková J., Hřebík Š. \& Kodrová Z. (2004a): Entomofauna a dlouhodobé trendy vývoje chemismu vody v tocích pramenné oblasti Otavy.- Aktuality šumavského výzkumu II, Vimperk: 192-196.

- (2004b): Macroinvertebrate communities and water quality in episodically acidified lotic ecosystems in the mountain region affected by bark beetle calamity.- Acta Universitatis Carolinae Environmentalica, 18: 3553.

Růžičková J. \& Kotrbová M. (2000): Aquatic entomocoenosis in lotic ecosystems of the upper Vydra basin (Šumava National Park, Czech Republic).- Silva Gabreta, 5: 135-148.

Soldán T. (1996): Přehled pošvatek Šumavy s poznámkami k jejich současnému výskytu (Plecoptera).Sborník Jihočeského Musea v Českých Budějovicích, Přírodní vědy, 36(1): 37-47.

- (2004): Aktualizovaný seznam pošvatek (Insecta, Plecoptera) Národního parku a Chráněné krajinné oblasti Šumava se zřetelem k jejich druhové ochrané.- Aktuality šumavského výzkumu II, Vimperk: 197-201.

Soldán T. \& Špaček J. (2002): Diversity of stoneflies (Plecoptera) of the Novohradské Mountains: A review of recent knowledge, pp. 205-209. In Papáček M. (ed.): Biodiverzita a př́rodní podmínky Novohradských hor. Jihočeská univerzita a Entomologický ústav AV ČR, České Budějovice, 285 pp.

Soldán T., Zahrádková S. \& Landa V. (2001): Dlouhodobé změny diverzity vodního hmyzu (Ephemeroptera, Plecoptera, Trichoptera) v regulovaném segmentu řeky Křemelné (Šumava, ČR).Aktuality šumavského výzkumu, Srní 2.-4. dubna 2001: 168-171.

Soldán T., Zahrádková S., Helešic J., Dušek L. \& Landa V. (1998) Distributional and Quantitative Patterns of Ephemeroptera and Plecoptera in the Czech Republic: A Possibility of Detection of Long-term 
Changes of Aquatic Biotopes. Folia Facultatis Scientiarum Naturalium Universitatis Masarykianae Brunensis, Brno, 98, 305 pp.

Sowa R. (1966): Brachyptera starmachi sp. n., plécoptère nouvellement trouve des Carpathes.- Bulletin de L'academie Polonaise des Sciences, Cl.II, 14(6): 427-432.

Straškraba M., Marvan P., Komárek J., Štěpánek M., Dvořáková M., Kulhavý V., Romanovský A., Ložek V., Landa V., Sýkora J., Říha P., Štys P., Lellák J., Frank S., Pekárková K., Hruška V., Lohnický K., Oliva O., Cihař J. \& Balon E. (1954): Sborník o fauně a flóře řek Lučiny a Morávky se zvláštním zřetelem ke stavbě přehrad na těchto řekách. Soubor prací kolektivu mladších pracovníků biologické fakulty Karlovy university v Praze, 559 pp.

Sukop I. \& Spurný P. (2003): Hyrobiological study of the Oslava river-- Acta universitatis agriculturae et silviculturae Mendelianae Brunensis, LI(4): 31-38.

Šámal J. (1920): Příspěvek k plecopterologickému a trichopterologickému výzkumu šumavských vod.Časopis Národního musea, Praha, 44: 114-116.

Špaček J. (1999): Pošvatky (Plecoptera) Krkonoš v letech 1996-1998.-- Opera Corcontica, 36: 163-169.

- (2001): A review of Stoneflies (Plecoptera) of the Krkonoše Mts.- Matthias Belivs Univ. Proc. 2001, 1(1): 4952.

Švec J. (1960): Příspěvek k poznání benthické fauny Vsetínské Bečvy.- Příroda Jihovýchodní Moravy 1, Oblastní muzeum Gottwaldov: 187-210.

Theischinger G. (1974): Plecoptera (Insecta) aus Oberösterreich, I. Rhabdiopteryx navicula spec. nov. (Taeniopterygidae) aus dem Innviertel.- Natur. Jahrbl. Stadt. Linz, 20: 185-194.

Teslenko V.A. \& Zhiltzova V.A. (2009): Opredelitel vesnyanok (Insecta, Plecoptera) Rossii i sopredelnykh stran. Imago i lichinki. Institute of Biology and Soil Science Far Eastern Branch of Russian Academy of Science, Vladivostok, Dalnauka, $382 \mathrm{pp}$.

Tierno de Figueroa J.M., Sánchez-Ortega A., Iglesia P.M. \& Luzón-Ortega J.M. (2003): Fauna Iberica Vol. 22. Plecoptera. Museo Nacional de Ciencias Naturales, Consejo Superior de Investigaciones Científicas, Madrid, 404 pp.

Tuša I. (1987): Struktura, dynamika a produkce zoobentosu pstruhového toku (Hučivá Desná, Hrubý Jeseník).Čas. Slez. Muz. Opava (A), 36: 193-218.

- 2001: K výskytu pošvatek (Plecoptera) na severní Moravě v letech 1975-2000. Závěrečná zpráva výzkumného úkolu, 22 pp.

Vinçon G. \& Murányi D. (2009): Revision of the Rhabdiopteryx neglecta species group (Plecoptera: Taniopterygidae).- Aquat. Insects, 31(suppl. 1): 203-218.

Weinzierl A. (1999): Nachweise von Isoperla albanica, Brachyptera starmachi und Brachyptera trifasciata (Plecoptera) aus Niederbayern.- Lauterbornia, 37: 19-23.

Winkler O. (1956): Výzkum bentické zvířeny potoků v okolí Horské Kvildy (Šumava).-Zoologické listy, 5: 367-386.

- (1963): Beitrag zur Kenntnis der Bodenfauna der oberen Moldau vor der Errichtung der Talsperre in Lipno.Acta Universitatis Carolinae - Biologica, 1: 85-101.

- (1977): Příspěvek k poznání potoční entomofauny v Krkonoších.- Opera Corcontica, 14: 143-153.

Zelinka M. (1950): K poznání zvířeny horských potoků Slezských Beskyd.- Zvláštní př́loha Př́rodovědeckého sborníku Ostravského kraje, XI(2-3): 3-28.

Zhiltzova V.A. (1997): Zoogeographic features of the Euhologhatha fauna (Plecoptera) of Russia and adjacent territories (within the limits of the former USSR), pp. 288-295. In Landolt P. \& Sartori M. (eds): Ephemeroptera \& Plecoptera: Biology-Ecology-Systematics. Maron+Tinguely \& Lachat SA, CH-Fribourg, $569 \mathrm{pp}$.

- (2003): Vesnyanki (Plecoptera). Gruppa Euholognatha. Fauna Rossii i sopredelnykh stran. Nauka, St. Petersburg, 537 pp.

Zwick P. (1992): Stream habitat fragmentation - a threat to biodiversity.- Biodivers. Conserv., 1: 80-97.

- (2004): Key to the West Palaearctic genera of stoneflies (Plecoptera) in the larval stage.- Limnologica, 34 : 315-348.

Authors' addresses: Jindřiška Bojková \& Michal Straka, Department of Botany and Zoology, Faculty of Science, Masaryk University, 2 Kotlářská, 61137 Brno, Czech Republic; e-mail: bojkova@centrum.cz; michal.straka@centrum.cz

Tomáš Soldán, Biology Centre, Academy of Sciences of the Czech Republic, Institute of Entomology, 31 Branišovská, 37005 České Budějovice, Czech Republic; e-mail: soldan@entu.cas.cz

Jan Špaček, Povodí Labe s.p., OVHL, 951 V. Nejedlého, 50003 Hradec Králové, Czech Republic; e-mail: spacek@pla.cz 